\title{
Checklist of the flower flies of Ecuador (Diptera, Syrphidae)
}

\author{
Diego Marín-Armijos', Noelia Quezada-Ríos', \\ Carolina Soto-Armijos', Ximo Mengual ${ }^{2}$
}

I Museo de Colecciones Biológicas, Departamento de Ciencias Biológicas, Universidad Técnica Particular de Loja, San Cayetano Alto s/n, C.P. 1101 608, Loja, Ecuador 2 Zoologisches Forschungsmuseum Alexander Koenig, Leibniz-Institut für Biodiversität der Tiere, Adenauerallee 160, D-53113 Bonn, Germany

Corresponding author: Ximo Mengual (x.mengual@leibniz-zfmk.de)

Academic editor: M. Hauser | Received 20 April 2017 | Accepted 2 June 2017 | Published 17 August 2017

http://zoobank.org/84A38A1F-CD07-45E5-AF47-6EFED63ACCD2

Citation: Marín-Armijos D, Quezada-Ríos N, Soto-Armijos C, Mengual X (2017) Checklist of the flower flies of Ecuador (Diptera, Syrphidae). ZooKeys 691: 163-199. https://doi.org/10.3897/zookeys.691.13328

\begin{abstract}
Syrphidae is one of the most speciose families of true flies, with more than 6,100 described species and worldwide distribution. They are important for humans acting as crucial pollinators, biological control agents, decomposers, and bioindicators. One third of its diversity is found in the Neotropical Region, but the taxonomic knowledge for this region is incomplete. Thus, taxonomic revisions and species checklists of Syrphidae in the Neotropics are the highest priority for biodiversity studies. Therefore, we present the first checklist of Syrphidae for Ecuador based on literature records, and provide as well the original reference for the first time species citations for the country. A total of 201 species were recorded for Ecuador, with more than 600 records from 24 provinces and 237 localities. Tungurahua, Pastaza, and Galápagos were the best sampled provinces. Although the reported Ecuadorian syrphid fauna only comprises 11.2 $\%$ of the described Neotropical species, Ecuador has the third highest flower fly diversity density after Costa Rica and Suriname. These data indicate the high species diversity for this country in such small geographic area.
\end{abstract}

\section{Keywords}

faunistics, hoverflies, Neotropical Region, species list, Syrphid fauna

Copyright Diego Marin-Armijos et al. This is an open access article distributed under the terms of the Creative Commons Attribution License (CC BY 4.0), which permits unrestricted use, distribution, and reproduction in any medium, provided the original author and source are credited. 


\section{Introduction}

Seventeen countries in the world are considered megadiverse, occupying less than $10 \%$ of the Earth's surface and comprising nearly $70 \%$ of the global biodiversity (Mittermeier et al. 2005). In this group, Ecuador is listed among the first places in the world ranking based on number of species per area unit for vascular plants, mammals, birds, reptiles and amphibians (Mittermeier and Mittermeier 1997, Brehm et al. 2008), but it is the megadiverse country with the smallest land area (Mittermeier et al. 2005). This makes Ecuador rank at the top, or near so, of the megadiversity list if diversity per unit area is taken into consideration. The geographic position of Ecuador and a series of biotic and abiotic elements have resulted in an extraordinary biological diversity in this nation. For instance, there are ca. 20,000 estimated plants in Ecuador, of which up to 5,000 are most likely endemics. In terms of vertebrate diversity, $1.3 \%$ of the global diversity of non-fish vertebrates is endemic to Ecuador (Mittermeier et al. 2005).

Insects are the most successful group of living organisms in our planet in number of species and different natural histories. From all known and described species on Earth, ca. 1.5-1.7 millions, between 65 and $75 \%$ are insects, and among the insect orders only four orders represent more than $80 \%$ of the species: Coleoptera, Lepidoptera, Diptera, and Hymenoptera (Adler and Foottit 2009). Despite being abundant and ubiquitous, insects are understudied in Ecuador and many new species and genera are waiting to be formally described (Dangles et al. 2009, Barragán et al. 2009). Furthermore, there is no estimate on the number of invertebrates for Ecuador, neither a checklist for most of the invertebrate groups present in this country (Salazar and Donoso 2014).

Diptera, which includes mosquitoes and true flies, comprises more than 153,000 described species (about 10-12\% of the planet's biodiversity) and has a rate of near 1,000 new species described per year (Pape and Thompson 2013). Diptera is not only rich in number of species, but also in morphology and structure, habitats use and human interactions (Courtney et al. 2009). Most recent studies in this country have focused mostly on Lepidoptera (Piñas and Manzano 1997, Bollino and Onore 2001, Piñas and Manzano 2003a, 2003b, Hilt and Fiedler 2005, Brehm 2005, Fiedler et al. 2007, Bodner et al. 2010), and Coleoptera (Celi et al. 2004, Moret 2005, Carpio et al. 2009, Carvajal et al. 2011, Domínguez et al. 2015, Thormann et al. 2016). Salazar and Donoso (2014) present some numbers for the invertebrate fauna of Ecuador and report 722 dipteran species from the literature, but this number is probably an underestimation, which was biased by the research focus of the previous works in Ecuador. Thus, the actual species number of species of Diptera for the country is far from being known.

Commonly called flower or hoverflies, the family Syrphidae is one of the most diverse in Diptera with more than 6,000 described species (Brown 2009), and the third taxon with most species in the Neotropical Region (Amorim 2009). Their coloration, from orange-brown in a few species to striking yellow and black patterns, causes them to be confused with bees or wasps (Hymenoptera). Adults of the family Syrphidae have the ability to hover and are associated with flowers, which are used as mating sites and food sources (pollen and nectar). Therefore, the imagoes are considered important pollinators of herbs, 
shrubs, and arboreal plants in natural ecosystems as well as in agricultural areas (Speight and Lucas 1992, Marinoni and Thompson 2003, Pérez-Bañón et al. 2003, Ssymank and Kearns 2009, Inouye et al. 2015). Syrphid species have been used as bioindicators as well to assess biodiversity loss and the efficiency of restoration and conservation policies (Sommaggio 1999, Tscharntke et al. 2005, Ricarte et al. 2011, Sommaggio and Burgio 2014).

Larvae are very variable in structure, habits, and feeding modes, including fungal fruiting bodies, brood in nests of social Hymenoptera, dung, decaying wood and water bodies of several types (Rotheray 1993, Rotheray and Gilbert 1999, 2011). Larvae of some species can mine leaves and stems of numerous plant families, or even feed on pollen, and others are predators of arthropods (aphids, caterpillars, larvae of flies or beetles, adult flies, etc.) or are kleptoparasitic or parasitoids (Rojo et al. 2003, Weng and Rotheray 2008, Reemer and Rotheray 2009, Rotheray et al. 2000, Ureña and Hanson 2010, Zuijen and Nishida 2011, Pérez-Lachaud et al 2014, Jordaens et al. 2015, Fleischmann et al. 2016, Dumbardon-Martial 2016). Due to their feeding mode, some syrphid species play an important role as biological control agents of pests (Greco 1998, Schmidt et al. 2004, Bergh and Short 2008, Bugg et al. 2008, Pineda and Marcos-García 2008, Nelson et al. 2012, Amorós-Jiménez et al. 2014, Eckberg et al. 2015) and as decomposers of organic matter (Lardé 1989, Rotheray et al. 2009, Martínez-Falcón et al. 2012).

Flower flies are distributed worldwide, with the exception of Antarctica and a few remote oceanic islands, and their greatest species diversity is found in the tropics (Vockeroth 1992, Reemer 2013, Reemer and Ståhls 2013b). The classification of Neotropical Syrphidae has been largely reviewed by Vockeroth (1969), Thompson (1972, 1999) and Reemer (2014), but the taxonomy of Syrphidae is far from being complete in the Neotropical Region yet, and many new species remain to be described (Mengual and Thompson 2008, Mengual et al. 2009, Thompson et al. 2010, Mengual 2011, Mengual and Thompson 2011, Reemer 2010, 2014, 2016). Moreover, the almost absence of identification keys for Neotropical species makes difficult the elaboration of regional checklists or the discovery of new species to science (Thompson et al. 2010, Montoya et al. 2012). Previously, species lists based on single surveys (Campos 1960, Linsley and Usinger 1966, Linsey 1977), for specific taxa (genus Toxomerus Macquart, 1855 by Gerdes, 1974a), or for limited areas, such as Galapagos Islands (Sinclair 2015, Sinclair et al. 2016), have been published for Ecuador.

Amorim (2009) considers Syrphidae among the still underexplored dipteran families in the Neotropical Region, and Ecuador among the most poorly collected areas in South America. Nevertheless, there have been some efforts during the last years to teach Syrphidae taxonomy to young students via workshops and courses with the great help of F.C. Thompson (USNM, National Museum of Natural History, Smithsonian Institution) as a coordinator (Colombia 2006, Peru 2008, Ecuador 2012), with the purpose to educate new taxonomists that may help to elucidate the thrilling evolutionary history of this group. As a fruit from these workshops, a strong collaboration among the authors was established years ago to study the flower flies of Ecuador. Currently, there is no species list for Ecuadorian flower flies that can help as a starting point, and the existing records are few and scattered thorough the literature. Conse- 
quently, a species checklist of the family Syrphidae in Ecuador for further biodiversity studies was the highest priority. In this survey, we present the first species checklist of Syrphidae for Ecuador based on literature records and provide as well the original reference for the first time species citations for the country of Ecuador.

\section{Materials and methods}

Thompson et al. (1976) was used as the primary source to check for species cited previously for Ecuador. Based on that keystone publication and Thompson et al. (2010), we reviewed all the published literature up to date in order to find references to Ecuadorian syrphids. Moreover, bibliographic searches were performed in public and scientific journal databases such as Google Scholar, Scopus, ISI Web of Knowledge, BioOne, Redalyc, Scielo, BioOne, ScienceDirect, and ResearchGate. Our keywords in English and Spanish for the searches were invertebrates, Ecuador, Diptera, Syrphidae, Neotropics, distribution, flower flies and hoverflies. In addition, we studied representative collections of Ecuador, i.e. Museo de Zoología de la Pontificia Universidad Católica del Ecuador (QCAZ) and Museo de la Escuela Politécnica Nacional.

To illustrate the flower fly records in a geographic map we used the coordinates available in the literature. For the localities without geographic coordinates we used Google Earth ${ }^{\circledR}$ to obtain them. Figure 1 was created using QGIS software (QGIS Development Team, 2009).

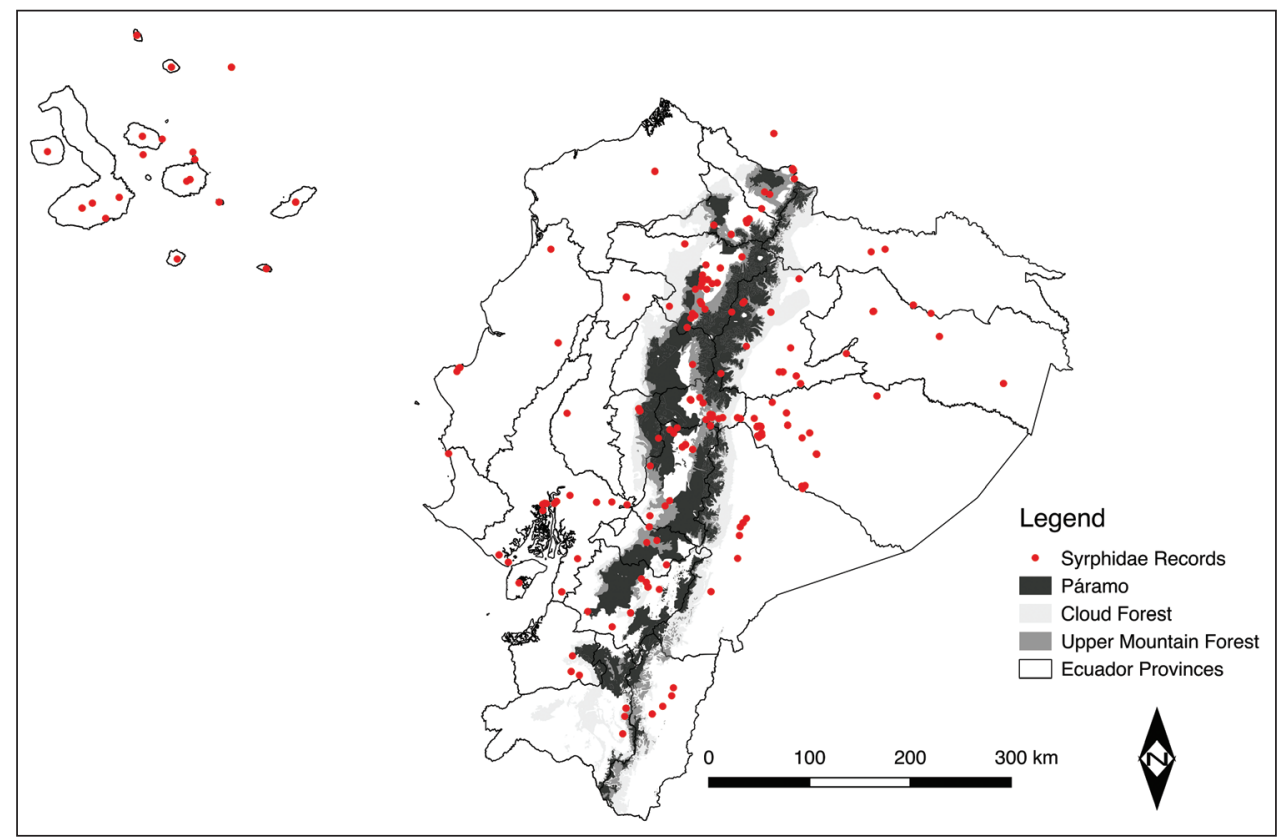

Figure I. Distribution map of flower fly records in Ecuador. 


\section{Results}

A total of 201 species plus four unidentified species and two misidentified taxa, belonging to 51 syrphid genera and subgenera, have been recorded up to date for Ecuador. More than 600 records from 24 provinces and 237 different localities of Ecuador are given in Table 1. Although there are records from all the Ecuadorian provinces, they do not show an even collecting effort for the whole country. Tungurahua (with 80 collecting events), Pastaza (72), and Galápagos (60) are the best sampled provinces, while the flower fly records for Orellana (3), Los Ríos (2), Santa Elena (2), Santo Domingo de Los Tsáchilas (2), and Esmeraldas (1) provinces are almost anecdotal. In terms of geographic Ecuadorian regions, the Sierra of Ecuador and the Galapagos Islands have been more extensively sampled and studied (Table 1 and Figure 1). On the other hand, the Costa Region, North and South Amazonia, and Austral Region of Ecuador have been little explored (Figure 1).

Table I. Checklist of Syrphidae species recorded from Ecuador, with the Ecuadorian province, locality, altitude (when cited in the original reference), and the original reference for Ecuador.

\begin{tabular}{|c|c|c|c|}
\hline Species & Province & $\begin{array}{c}\text { Locality } \\
\text { (Altitude masl) } \\
\end{array}$ & References for Ecuador \\
\hline $\begin{array}{l}\text { Alipumilio femoratus } \\
\text { Shannon, } 1927\end{array}$ & Pastaza & Puyo & Rotheray et al. 2000: 137 \\
\hline $\begin{array}{l}\text { Allograpta annulipes } \\
\text { (Macquart, 1850) }\end{array}$ & Pastaza & $\begin{array}{l}\text { Santa Inés, Río } \\
\text { Pastaza (1200) }\end{array}$ & Fluke 1942: 16 (as A. geminata) \\
\hline Allograpta browni Fluke, 1942 & Imbabura & Cuicocha (3200) & Fluke 1942: 18 \\
\hline \multirow{3}{*}{$\begin{array}{l}\text { Allograpta exotica } \\
\text { (Wiedemann, 1830) }\end{array}$} & Tungurahua & Baños & Fluke 1942: 19 \\
\hline & Tungurahua & Juive & Fluke 1942: 19 \\
\hline & Ecuador & & Fluke 1950a: 146 (as Syrphus exoticus) \\
\hline \multirow{2}{*}{ Allograpta falcata Fluke, 1942} & Tungurahua & Baños (1800) & Fluke 1942: 16 \\
\hline & Tungurahua & Baños (2200) & Fluke 1942: 16 \\
\hline \multirow{12}{*}{$\begin{array}{l}\text { Allograpta neosplendens Sinclair \& } \\
\text { Thompson, } 2016\end{array}$} & Galápagos & Española & Sinclair and Peck 2002; Sinclair et al. 2016: 87 \\
\hline & Galápagos & Fernandina & Sinclair et al. 2016: 87 \\
\hline & Galápagos & Floreana & $\begin{array}{l}\text { Coquillett 1901: 374; Linsley and Usinger } \\
\text { 1966: 168; Linsley 1977: 39; Sinclair and Peck } \\
\text { 2002; Sinclair 2015; Sinclair et al. 2016: } 87\end{array}$ \\
\hline & Galápagos & Genovesa & Sinclair et al. 2016: 87 \\
\hline & Galápagos & Isabela & $\begin{array}{l}\text { Sinclair and Peck 2002; Sinclair 2015; Sinclair } \\
\text { et al. 2016: } 87\end{array}$ \\
\hline & Galápagos & Marchena & Sinclair 2015 \\
\hline & Galápagos & Pinta & Sinclair and Peck 2002; Sinclair et al. 2016: 87 \\
\hline & Galápagos & San Cristóbal & $\begin{array}{l}\text { Curran 1934: 153; Linsley and Usinger 1966: } \\
\text { 168; Linsley 1977: 39; Sinclair and Peck 2002; } \\
\text { Sinclair et al. 2016: } 87\end{array}$ \\
\hline & Galápagos & Santa Cruz & $\begin{array}{l}\text { Boada 2005: 84; Sinclair 2015; Sinclair et al. } \\
\text { 2016: } 87\end{array}$ \\
\hline & Galápagos & Santa Fé & Sinclair 2015 \\
\hline & Galápagos & Santiago & $\begin{array}{l}\text { Coquillett 1901: 374; Curran 1934: 153; } \\
\text { Linsley and Usinger 1966: 168; Linsley 1977: } \\
\text { 39; Sinclair and Peck 2002; Sinclair et al. } \\
\text { 2016: } 87\end{array}$ \\
\hline & Galápagos & & Thomson 1869: 501 (as Syrphus splendens) \\
\hline
\end{tabular}




\begin{tabular}{|c|c|c|c|}
\hline Species & Province & $\begin{array}{c}\text { Locality } \\
\text { (Altitude masl) }\end{array}$ & References for Ecuador \\
\hline \multirow{2}{*}{ Allograpta neotropica Curran, 1936} & Tungurahua & Baños & Fluke 1942: 20 \\
\hline & Pastaza & Santa Inés & Fluke 1942: 20 \\
\hline \multirow{3}{*}{ Allograpta obliqua (Say, 1823) } & Tungurahua & Baños & Fluke 1942: 19 \\
\hline & Carchi & Tulcán & Campos 1960: 25 \\
\hline & Chimborazo & Riobamba & Campos 1960: 25 \\
\hline \multirow{3}{*}{ Allograpta tectiforma Fluke, 1942} & Imbabura & Cuicocha (3200) & Fluke 1942: 19 \\
\hline & Imbabura & Cuicocha $(3500)$ & Fluke 1942: 19 \\
\hline & Ecuador & & Fluke 1950a: 146 \\
\hline Allograpta teligera Fluke, 1942 & Tungurahua & Bańos (1800) & Fluke 1942: 18 \\
\hline \multirow{6}{*}{$\begin{array}{l}\text { Argentinomyia agonis } \\
\text { (Walker, 1849) }\end{array}$} & Galápagos & & Walker 1849: 588; Linsley 1977: 39 \\
\hline & Galápagos & Floreana & Sinclair and Peck 2002; Sinclair 2015 \\
\hline & Galápagos & Isabela & Sinclair and Peck 2002; Sinclair et al. 2016: 85 \\
\hline & Galápagos & Pinta & Sinclair and Peck 2002; Sinclair et al. 2016: 85 \\
\hline & Galápagos & San Cristóbal & Sinclair 2015 \\
\hline & Galápagos & Santa Cruz & $\begin{array}{l}\text { Boada 2005: 86; Sinclair 2015; Sinclair et al. } \\
\text { 2016: } 85\end{array}$ \\
\hline \multirow{2}{*}{$\begin{array}{l}\text { Argentinomyia altissima } \\
\text { (Fluke, 1945) }\end{array}$} & Imbabura & Cuicocha (3200) & Fluke 1945: 20 \\
\hline & Ecuador & & Fluke 1958: 266 \\
\hline \multirow{2}{*}{$\begin{array}{l}\text { Argentinomyia bolivariensis } \\
\text { (Fluke, 1945) }\end{array}$} & Bolívar & $\begin{array}{l}\text { Hda. Talahua } \\
(3100)\end{array}$ & Fluke 1945: 19 \\
\hline & Ecuador & & Fluke 1958: 266 \\
\hline \multirow{2}{*}{$\begin{array}{l}\text { Argentinomyia browni } \\
\text { (Fluke, 1945) }\end{array}$} & Bolívar & $\begin{array}{l}\text { Hda. Talahua } \\
(3100)\end{array}$ & Fluke 1945: 19 \\
\hline & Ecuador & & Fluke 1958: 266 \\
\hline Argentinomyia festiva (Fluke, 1945) & Tungurahua & Baños (1800) & Fluke 1945: 10 \\
\hline $\begin{array}{l}\text { Argentinomyia longicornis } \\
\text { (Walker, 1836) }\end{array}$ & Pastaza & Puyo (3000) & Fluke 1945: 4 \\
\hline \multirow{2}{*}{$\begin{array}{l}\text { Argentinomyia luculenta } \\
\text { (Fluke, 1945) }\end{array}$} & Tungurahua & Bańos (2300) & Fluke 1945: 18 \\
\hline & Tungurahua & Pondoa (2800) & Fluke 1945: 18 \\
\hline \multirow{2}{*}{ Argentinomyia opaca (Fluke, 1945) } & Chimborazo & Urbina $(3650)$ & Fluke 1945: 11 \\
\hline & Ecuador & & Fluke 1958: 266 \\
\hline \multirow[t]{2}{*}{ Argentinomyia rex (Fluke, 1945) } & Bolívar & $\begin{array}{l}\text { Hda. Talahua } \\
(3100)\end{array}$ & Fluke 1945: 22 \\
\hline & Ecuador & & Fluke 1958: 266 \\
\hline $\begin{array}{l}\text { Argentinomyia tropica } \\
\text { (Curran, 1937) }\end{array}$ & Tungurahua & Baños (2300) & Fluke 1945: 17 \\
\hline \multirow{2}{*}{$\begin{array}{l}\text { Claraplumula latifacies } \\
\text { Shannon, } 1927\end{array}$} & Bolívar & $\begin{array}{l}\text { Hda. Talahua } \\
(3100)\end{array}$ & Fluke 1942: 4 \\
\hline & Ecuador & & Fluke 1950a: 146 \\
\hline $\begin{array}{l}\text { Copestylum }(\text { Copestylum }) \\
\text { otongaensis Rotheray \& } \\
\text { Hancock, } 2007\end{array}$ & Cotopaxi & Otonga & Rotheray et al. 2007: 290 \\
\hline $\begin{array}{l}\text { Copestylum (Copestylum) tapia } \\
\text { Rotheray \& Hancock, } 2007\end{array}$ & Cotopaxi & Otonga & Rotheray et al. 2007: 307 \\
\hline $\begin{array}{l}\text { Copestylum (Phalacromya) } \\
\text { araceorum Ricarte } \& \\
\text { Rotheray, } 2015 \\
\end{array}$ & Cotopaxi & Otonga & Ricarte et al. 2015: 13 \\
\hline \multirow{4}{*}{$\begin{array}{l}\text { Copestylum (Phalacromya) beatricea } \\
\text { (Hull, 1950) }\end{array}$} & Azuay & Cuenca (2650) & Fluke 1951b: 15 (as Volucella ecuadorea) \\
\hline & Tungurahua & Baños & Fluke 1951b: 15 (as Volucella ecuadorea) \\
\hline & Imbabura & Cuicocha $(3300)$ & Fluke 1951b: 15 (as Volucella ecuadorea) \\
\hline & Ecuador & & Hull 1950: 236 \\
\hline
\end{tabular}




\begin{tabular}{|c|c|c|c|}
\hline Species & Province & $\begin{array}{c}\text { Locality } \\
\text { (Altitude masl) } \\
\end{array}$ & References for Ecuador \\
\hline \multirow{2}{*}{$\begin{array}{l}\text { Copestylum (Phalacromya) } \\
\text { brunneum (Thunberg, 1789) }\end{array}$} & Chimborazo & Huigra & Campos 1960: 27 (as Volucella esuriens) \\
\hline & Cañar & Azogues & Campos 1960: 27 (as Volucella esuriens) \\
\hline $\begin{array}{l}\text { Copestylum (Phalacromya) } \\
\text { bulbosum (Fluke, 1951) }\end{array}$ & Tungurahua & $\begin{array}{l}\text { Minza Chica } \\
(3750)\end{array}$ & Fluke 1951b: 25 \\
\hline $\begin{array}{l}\text { Copestylum (Phalacromya) camposi } \\
\text { (Curran, 1939) }\end{array}$ & Guayas & $\begin{array}{l}\text { Isla Puná, Puerto } \\
\text { Grande }\end{array}$ & Curran 1939: 8 \\
\hline \multirow{2}{*}{$\begin{array}{l}\text { Copestylum (Phalacromya) } \\
\text { chaetophorum (Williston, 1887) }\end{array}$} & Guayas & San Rafael & Campos 1960: 27 \\
\hline & Guayas & Bucay & Campos 1960: 27 \\
\hline \multirow{4}{*}{$\begin{array}{l}\text { Copestylum (Phalacromya) currani } \\
\text { (Fluke, 1951) }\end{array}$} & Pichincha & Guayllabamba & Rotheray et al. 2009: 714 \\
\hline & Tungurahua & Baños & Fluke 1951b: 13 \\
\hline & Imbabura & Cuicocha (3300) & Fluke 1951b: 13 \\
\hline & Azuay & Cuenca $(2650)$ & Fluke 1951b: 13 \\
\hline \multirow{3}{*}{$\begin{array}{l}\text { Copestylum (Phalacromya) } \\
\text { fulvicorne (Bigot, 1883) }\end{array}$} & Guayas & San Eduardo & Campos 1960: 27 \\
\hline & Guayas & Durán & Campos 1960: 27 \\
\hline & Guayas & San Rafael & Campos 1960: 27 \\
\hline $\begin{array}{l}\text { Copestylum (Phalacromya) } \\
\text { hambletoni (Fluke, 1951) }\end{array}$ & Ecuador & & Thompson et al. 1976: 77 \\
\hline $\begin{array}{l}\text { Copestylum (Phalacromya) } \\
\text { multipunctatum Rotheray \& } \\
\text { Hancock, } 2009\end{array}$ & Pichincha & Guayllabamba & Rotheray et al. 2009: 704 \\
\hline $\begin{array}{l}\text { Copestylum (Phalacromya) nigripes } \\
\text { (Bigot, 1857) }\end{array}$ & El Oro & Chillacocha & Campos 1960: 29 (as Phalacromyia concolor) \\
\hline $\begin{array}{l}\text { Copestylum (Phalacromya) placivum } \\
\text { (Hull, 1943) }\end{array}$ & Pastaza & Santa Inés & Hull 1943b: 31 \\
\hline $\begin{array}{l}\text { Copestylum (Phalacromya) } \\
\text { rufoscutellare (Philippi, 1865) }\end{array}$ & Chimborazo & Mirador & Campos 1960: 29 \\
\hline \multirow{2}{*}{$\begin{array}{l}\text { Copestylum (Phalacromya) } \\
\text { scintillans (Hull, 1949) }\end{array}$} & Galápagos & $\begin{array}{l}\text { San Cristóbal } \\
(730)\end{array}$ & Sinclair et al. 2016: 83 \\
\hline & Galápagos & Santa Cruz & Sinclair 2015 (as C. cf. viridana) \\
\hline \multirow{3}{*}{$\begin{array}{l}\text { Copestylum (Phalacromya) sica } \\
\text { (Curran, 1953) }\end{array}$} & Pichincha & Guayllabamba & Rotheray et al. 2009: 720 \\
\hline & \begin{tabular}{|l|} 
Tungurahua \\
\end{tabular} & Bańos & Curran 1953: 9 \\
\hline & Azuay & Tarqui & Curran 1953: 9 \\
\hline \multirow{4}{*}{$\begin{array}{l}\text { Copestylum (Phalacromya) splendens } \\
\text { (Townsend, 1897) }\end{array}$} & Pichincha & Cotocollao & Campos 1960: 27 (as Volucella opalina) \\
\hline & Tungurahua & Ambato & Campos 1960: 27 (as Volucella opalina) \\
\hline & Loja & Loja & Campos 1960: 27 (as Volucella opalina) \\
\hline & Chimborazo & Riobamba & Campos 1960: 27 (as Volucella opalina) \\
\hline $\begin{array}{l}\text { Copestylum (Phalacromya) } \\
\text { viridigaster (Hull, 1943) }\end{array}$ & Ecuador & & Hull 1943h: 41 \\
\hline $\begin{array}{l}\text { Dasysyrphus aff. lotus } \\
\text { (Williston, 1887) }\end{array}$ & Pichincha & Pichincha (3300) & Fluke 1942: 3 \\
\hline $\begin{array}{l}\text { Dolichogyna chilensis } \\
\text { (Walker, 1836) }\end{array}$ & Azuay & Narihuin̄a & Campos 1960: 29 \\
\hline \multirow{2}{*}{ Dolichogyna mulleri Fluke, 1951} & Azuay & Girón & Fluke 1951a: 472 \\
\hline & Imbabura & Cuicocha (3200) & Fluke 1951a: 472 \\
\hline $\begin{array}{l}\text { Eosalpingogaster nigriventris } \\
\text { (Bigot, 1883) }\end{array}$ & Guayas & $\begin{array}{l}\text { Isla Puná, Puerto } \\
\text { Grande (253) }\end{array}$ & Fluke 1937: 11 (as Salpingogaster liposeta) \\
\hline \multirow{4}{*}{$\begin{array}{l}\text { Eristalis (Eoseristalis) bogotensis } \\
\text { Macquart, } 1842\end{array}$} & Ecuador & & Thompson et al. 1976: 101 \\
\hline & \begin{tabular}{|l|} 
Napo- \\
Pichincha
\end{tabular} & $\begin{array}{l}\text { Antisamilla to } \\
\text { Pinatura }(3350)\end{array}$ & Thompson 1997: 223 \\
\hline & Pichincha & $\begin{array}{l}\text { Santa Catalina } \\
\text { Expt. Station } \\
(2780) \\
\end{array}$ & Thompson 1997: 223 \\
\hline & Pichincha & Quito (2850) & Thompson 1997: 223 \\
\hline
\end{tabular}




\begin{tabular}{|c|c|c|c|}
\hline Species & Province & $\begin{array}{c}\text { Locality } \\
\text { (Altitude masl) } \\
\end{array}$ & References for Ecuador \\
\hline \multirow{13}{*}{$\begin{array}{l}\text { Eristalis (Eoseristalis) bogotensis } \\
\text { Macquart, } 1842\end{array}$} & Chimborazo & 8 mi NE of Tixan & Thompson 1997: 223 \\
\hline & Chimborazo & Lago Zurucuchu & Thompson 1997: 223 \\
\hline & Tungurahua & Ambato (2700) & Thompson 1997: 223 \\
\hline & Carchi & Troya & Thompson 1997: 223 \\
\hline & Carchi & Tulcan (2800) & Thompson 1997: 223 \\
\hline & Carchi & El Ángel (2700) & Thompson 1997: 223 \\
\hline & Cañar & El Tambo (2800) & Thompson 1997: 223 \\
\hline & Pichincha & Pomasqui (2200) & Thompson 1997: 223 \\
\hline & Pichincha & $\begin{array}{l}\text { Valle de Machachi } \\
(2900)\end{array}$ & Thompson 1997: 223 \\
\hline & Loja & Loja $(2500)$ & Thompson 1997: 223 \\
\hline & Azuay & Tarqui (2800) & Thompson 1997: 223 \\
\hline & Azuay & $\begin{array}{l}28 \mathrm{~km} \mathrm{~S} \text { of Cuenca } \\
(2500-2800)\end{array}$ & Thompson 1997: 223 \\
\hline & Azuay & Cuenca (2200) & Thompson 1997: 223 \\
\hline \multirow{8}{*}{$\begin{array}{l}\text { Eupeodes (Metasyrphus) rojasi } \\
\text { Marneff, } 1999\end{array}$} & Azuay & $\begin{array}{l}\text { Gualaduisa Road } \\
(2150)\end{array}$ & Thompson 1999: 339 \\
\hline & Tungurahua & Baños & Thompson 1999: 339 \\
\hline & Bolívar & $\begin{array}{l}\text { Chota River, } \\
\text { Carchi (2000) } \\
\end{array}$ & Thompson 1999: 339 \\
\hline & Imbabura & $\begin{array}{l}\text { NW Ibarra, } \\
\text { Taguando River } \\
(1650-1900)\end{array}$ & Thompson 1999: 339 \\
\hline & Carchi & El Ángel (2700) & Thompson 1999: 339 \\
\hline & Carchi & $\begin{array}{l}10 \text { km SW Tulcán } \\
(2900)\end{array}$ & Thompson 1999: 339 \\
\hline & Imbabura & $\begin{array}{l}3 \mathrm{~km} \text { N Ibarra, } \\
\text { Yaguarcocha } \\
(1950)\end{array}$ & Thompson 1999: 339 \\
\hline & Pichincha & $\begin{array}{l}\text { Pichincha, } 2 \mathrm{~km} \mathrm{~W} \\
\text { Cayambe }(2300)\end{array}$ & Thompson 1999: 339 \\
\hline \multirow{3}{*}{ Fazia alta (Curran, 1936) } & Tungurahua & Baños & Fluke 1942: 14 \\
\hline & Tungurahua & Juive & Fluke 1942: 14 \\
\hline & Ecuador & & Fluke 1950a: 146 \\
\hline \multirow{11}{*}{ Fazia altissima (Fluke, 1942) } & Tungurahua & $\begin{array}{l}\text { Volcán } \\
\text { Tungurahua, } \\
\text { Minza Chica } \\
(3200)\end{array}$ & Fluke 1942: 10 \\
\hline & Tungurahua & Pondoa (2800) & Fluke 1942: 10 \\
\hline & Pichincha & $\begin{array}{l}\text { Páramo del Cerro, } \\
\text { Pasochoa (3300) }\end{array}$ & Fluke 1942: 10 \\
\hline & Pichincha & $\begin{array}{l}\text { Hda. San Rafael, } \\
\text { Río San Pedro } \\
(2700)\end{array}$ & Fluke 1942: 10 \\
\hline & Pichincha & $\begin{array}{l}\text { Uyumbicho } \\
(2650)\end{array}$ & Fluke 1942: 10 \\
\hline & Pichincha & $\begin{array}{l}\text { Hda. San Rafael } \\
(3000)\end{array}$ & Fluke 1942: 10 \\
\hline & Imbabura & Cuicocha (3200) & Fluke 1942: 10 \\
\hline & Ecuador & & Fluke 1950a: 145 \\
\hline & Pastaza & Puyo (1000) & Fluke 1942: 14 \\
\hline & Pichincha & $\begin{array}{l}\text { Uyumbicho } \\
(2700)\end{array}$ & Fluke 1942: 14 \\
\hline & Imbabura & Cuicocha (3200) & Fluke 1942: 14 \\
\hline
\end{tabular}




\begin{tabular}{|c|c|c|c|}
\hline Species & Province & $\begin{array}{c}\text { Locality } \\
\text { (Altitude masl) } \\
\end{array}$ & References for Ecuador \\
\hline \multirow{6}{*}{ Fazia argentipila (Fluke, 1942) } & Tungurahua & $\begin{array}{l}\text { Bańos, Runtun } \\
\text { trail }(2100)\end{array}$ & Fluke 1942: 14 \\
\hline & Pichincha & $\begin{array}{l}\text { Hda. San Rafael } \\
(3000)\end{array}$ & Fluke 1942: 14 \\
\hline & Azuay & Cuenca $(2500)$ & Fluke 1942: 14 \\
\hline & Tungurahua & $\begin{array}{l}\text { Baños, San Pablo } \\
(2200)\end{array}$ & Fluke 1942: 14 \\
\hline & Bolívar & $\begin{array}{l}\text { Hda. Talahua } \\
(3100)\end{array}$ & Fluke 1942: 14 \\
\hline & Ecuador & & Fluke 1950a: 145 \\
\hline \multirow{5}{*}{ Fazia colombia (Curran, 1925) } & Tungurahua & Baños (2100) & Fluke 1942: 13 \\
\hline & Azuay & Cuenca (2500) & Fluke 1942: 13 \\
\hline & Pichincha & Pichincha (2700) & Fluke 1942: 13 \\
\hline & $\begin{array}{l}\text { Morona } \\
\text { Santiago } \\
\end{array}$ & Sucúa (900) & Fluke 1942: 13 \\
\hline & Ecuador & & Fluke 1950a: 146 \\
\hline $\begin{array}{l}\text { Fazia decemmaculata } \\
\text { (Shannon, 1927) }\end{array}$ & Ecuador & & $\begin{array}{l}\text { Thompson et al. 1976: } 34 \text { (as F. bullaephora); } \\
\text { Mengual et al. 2009: } 17 \text { (as F. bullaephora) }\end{array}$ \\
\hline \multirow{4}{*}{ Fazia fasciata (Curran, 1932) } & Tungurahua & Juive (1900) & Fluke 1942: 13 \\
\hline & Tungurahua & Baños (1900) & Fluke 1942: 13 \\
\hline & Imbabura & Cuicocha (3200) & Fluke 1942: 13 \\
\hline & Ecuador & & Fluke 1950a: 146 \\
\hline \multirow{2}{*}{ Fazia fascifrons (Macquart, 1846) } & Bolívar & $\begin{array}{l}\text { Hda. Talahua } \\
(3100)\end{array}$ & Fluke 1942: 12 (as Epistrophe armillata) \\
\hline & Ecuador & & Fluke 1950a: 145 (as Epistrophe armillatus) \\
\hline Fazia imitator (Curran, 1925) & Tungurahua & $\begin{array}{l}\text { Río Mapoto } \\
(1400)\end{array}$ & Fluke 1942: 11 \\
\hline \multirow[t]{3}{*}{ Fazia luna (Fluke, 1942) } & Tungurahua & $\begin{array}{l}\text { Volcán } \\
\text { Tungurahua, } \\
\text { Minza Chica } \\
(3200)\end{array}$ & Fluke 1942: 8 \\
\hline & Bolívar & \begin{tabular}{|l} 
Hda. Talahua \\
$(3100)$
\end{tabular} & Fluke 1942: 8 \\
\hline & Ecuador & & Fluke 1950a: 146 \\
\hline \multirow{3}{*}{$\begin{array}{l}\text { Fazia micrura (Osten } \\
\text { Sacken, 1877) }\end{array}$} & $\begin{array}{l}\text { Morona } \\
\text { Santiago }\end{array}$ & Sucúa & Fluke 1942: 14 \\
\hline & $\begin{array}{l}\text { Morona } \\
\text { Santiago }\end{array}$ & Macas & Fluke 1942: 14 \\
\hline & Carchi & Tulcán & Campos 1960: 26 (as Sphaerophoria picticauda) \\
\hline \multirow[t]{3}{*}{ Fazia remigis (Fluke, 1942) } & Tungurahua & \begin{tabular}{|l|} 
Volcán \\
Tungurahua, \\
Minza Chica \\
$(3200)$ \\
\end{tabular} & Fluke 1942: 9 \\
\hline & Bolívar & $\begin{array}{l}\text { Hda. Talahua } \\
(3100)\end{array}$ & Fluke 1942: 9 \\
\hline & Ecuador & & Fluke 1950a: 145 \\
\hline \multirow[t]{2}{*}{ Fazia roburoris (Fluke, 1942) } & Bolívar & $\begin{array}{l}\text { Hda. Talahua } \\
(3100)\end{array}$ & Fluke 1942: 11 \\
\hline & Ecuador & & Fluke 1950a: 146 \\
\hline $\begin{array}{l}\text { Hypselosyrphus marshalli } \\
\text { Reemer, } 2013\end{array}$ & Napo & $\begin{array}{l}\text { Tiputini } \\
\text { Diodiversity } \\
\text { Station } \\
\end{array}$ & Reemer 2013: 28 \\
\hline Leucopodella boadicea (Hull, 1943) & El Oro & Piñas (1506) & Hull 1943i: 73 \\
\hline $\begin{array}{l}\text { Leucopodella delicatula } \\
\text { (Hull, 1943) }\end{array}$ & Tungurahua & Bańos & Hull 1943i: 78 \\
\hline
\end{tabular}




\begin{tabular}{|c|c|c|c|}
\hline Species & Province & $\begin{array}{c}\text { Locality } \\
\text { (Altitude masl) } \\
\end{array}$ & References for Ecuador \\
\hline $\begin{array}{l}\text { Leucopodella gracilis } \\
\text { (Williston, 1891) }\end{array}$ & Ecuador & & Thompson et al. 1976: 46 (as L. asthenia) \\
\hline Leucopodella zenilla (Hull, 1943) & Bolívar & $\begin{array}{l}\text { Hda. Talahua } \\
(3100)\end{array}$ & Hull 1943g: 77 \\
\hline Lycastrirhyncha nitens Bigot, 1859 & Ecuador & & $\begin{array}{l}\text { Montoya et al. 2012: supplementary material } \\
\text { page 3; Montoya et al. 2016: } 492\end{array}$ \\
\hline Mallota aberrans Shannon, 1927 & Napo & $\begin{array}{l}7 \mathrm{~km} \mathrm{~S} \text { of Baeza } \\
(2000)\end{array}$ & Thompson and Zumbado 2002: 93 \\
\hline Mallota nigra Shannon, 1927 & Pastaza & Santa Inés & Shannon 1927: 17 \\
\hline Mallota rubicunda Curran, 1940 & Tungurahua & $\begin{array}{l}\text { Volcán } \\
\text { Tungurahua } \\
(2600) \\
\end{array}$ & Curran 1940: 13 \\
\hline $\begin{array}{l}\text { Meromacrus laconicus } \\
\text { (Walker, 1852) }\end{array}$ & Guayas & Isla Puná (253) & Blatch et al. 2003: 26 \\
\hline $\begin{array}{l}\text { Meromacrus panamensis } \\
\text { Curran, } 1930\end{array}$ & Guayas & San Eduardo & Campos 1960: 29 \\
\hline $\begin{array}{l}\text { Meromacrus pratorum } \\
\text { (Fabricius, 1775) }\end{array}$ & Ecuador & & Thompson et al. 1976: 113 \\
\hline Meropidia rufa Thompson, 1983 & $\begin{array}{l}\text { Morona } \\
\text { Santiago }\end{array}$ & $\begin{array}{l}\text { Limón Indanza } \\
(900)\end{array}$ & Hippa and Thompson, 1983: 110 \\
\hline $\begin{array}{l}\text { Microdon (Chymophila) fulgens } \\
\text { Wiedemann, } 1830\end{array}$ & Guayas & $\begin{array}{l}\text { Guayaquil, San } \\
\text { Eduardo }\end{array}$ & Campos 1960: 24 \\
\hline $\begin{array}{l}\text { Microdon (Microdon) violaceus } \\
\text { (Macquart, 1842) }\end{array}$ & Guayas & Durán & Campos 1960: 24 \\
\hline Microdon sp. & Guayas & env. of Guayaquil & Campos 1960: 24 \\
\hline Mixogaster thecla (Hull, 1954) & Ecuador & & Thompson et al. 1976: 59 \\
\hline $\begin{array}{l}\text { Ocyptamus (Calostigma) elnora } \\
\text { (Shannon, 1927) }\end{array}$ & Ecuador & & Thompson et al. 1976: 17 \\
\hline \multirow{2}{*}{$\begin{array}{l}\text { Ocyptamus (Hermesomyia) } \\
\text { wulpianus (Lynch } \\
\text { Arribalzaga, 1891) }\end{array}$} & Pastaza & Puyo (1250) & Hull 1943a: 50 (as Baccha phobifer) \\
\hline & Pichincha & $\begin{array}{l}40 \text { km SW Quito, } \\
\text { Tandapi (1300- } \\
1500)\end{array}$ & $\begin{array}{l}\text { Vockeroth 1969: } 123 \text { (as Hermesomyia } \\
\text { bacchiformis) }\end{array}$ \\
\hline $\begin{array}{l}\text { Ocyptamus (Hybobathus) } \\
\text { flavipennis (Wiedemann, 1830) }\end{array}$ & Ecuador & & Thompson et al. 1976: 18 \\
\hline \multirow{2}{*}{$\begin{array}{l}\text { Ocyptamus (Mimocalla) bonariensis } \\
\text { (Curran, 1941) }\end{array}$} & Tungurahua & Bańos & Curran 1941: 284 (as Salpingogaster flukei) \\
\hline & Tungurahua & Baños, Chaupi & Hull 1943a: 51 (as Baccha phobia) \\
\hline $\begin{array}{l}\text { Ocyptamus (Ocyptamus) aeolus } \\
\text { (Hull, 1943) }\end{array}$ & Pastaza & $\begin{array}{l}\text { Machai, Río } \\
\text { Pastaza }(1300)\end{array}$ & Hull 1943g: 70 \\
\hline $\begin{array}{l}\text { Ocyptamus (Ocyptamus) anonus } \\
\text { (Hull, 1943) }\end{array}$ & Pastaza & Puyo (1000) & Hull 1943d: 91 \\
\hline $\begin{array}{l}\text { Ocyptamus (Ocyptamus) cultratus } \\
\text { (Austen, 1893) }\end{array}$ & Manabí & Palmar & Hull 1943g: 78 (as Baccha satyra) \\
\hline $\begin{array}{l}\text { Ocyptamus (Ocyptamus) cymbellina } \\
\text { (Hull, 1944) }\end{array}$ & $\begin{array}{l}\text { Santo } \\
\text { Domingo de } \\
\text { los Tsáchilas } \\
\end{array}$ & $\begin{array}{l}\text { Santo Domingo } \\
(950)\end{array}$ & Hull 1944b: 64 \\
\hline \multirow{2}{*}{$\begin{array}{l}\text { Ocyptamus (Ocyptamus) dimidiatus } \\
\text { (Fabricius, 1781) }\end{array}$} & Guayas & $\begin{array}{l}\text { Guayaquil, San } \\
\text { Eduardo }\end{array}$ & Campos 1960: 24 \\
\hline & Guayas & San Eduardo & Campos 1960: 24 \\
\hline $\begin{array}{l}\text { Ocyptamus (Ocyptamus) niobe } \\
\text { (Hull, 1943) }\end{array}$ & Manabí & Palmar (200) & Hull 1943i: 74 \\
\hline $\begin{array}{l}\text { Ocyptamus (Ocyptamus) princeps } \\
\text { (Hull, 1944) }\end{array}$ & Pastaza & Puyo (1000) & Hull 1944b: 57 \\
\hline $\begin{array}{l}\text { Ocyptamus (Ocyptamus) saffrona } \\
\text { (Hull, 1943) }\end{array}$ & Manabí & Palmar & Hull 1943i: 74 \\
\hline $\begin{array}{l}\text { Ocyptamus (Ocyptamus) zilla } \\
\text { (Hull, 1943) }\end{array}$ & Pastaza & Puyo & Hull 1943j: 215 \\
\hline
\end{tabular}




\begin{tabular}{|c|c|c|c|}
\hline Species & Province & $\begin{array}{c}\text { Locality } \\
\text { (Altitude masl) } \\
\end{array}$ & References for Ecuador \\
\hline $\begin{array}{l}\text { Ocyptamus (Orphnabaccha) } \\
\text { cerberus (Hull, 1943) }\end{array}$ & Imbabura & Cuicocha & Hull 1943g: 67 \\
\hline \multirow{3}{*}{$\begin{array}{l}\text { Ocyptamus (Orphnabaccha) opacus } \\
\text { (Fluke, 1950) }\end{array}$} & Tungurahua & $\begin{array}{l}\text { Volcán } \\
\text { Tungurahua } \\
(2800) \\
\end{array}$ & Fluke 1950b: 444 \\
\hline & Tungurahua & Ваn̆os (1900) & Fluke 1950b: 444 \\
\hline & $\begin{array}{l}\text { Napo- } \\
\text { Orellana } \\
\end{array}$ & $\begin{array}{l}\text { Sumaco [as Río } \\
\text { Zumac] (1400) }\end{array}$ & Fluke 1950b: 444 \\
\hline \multirow[t]{3}{*}{$\begin{array}{l}\text { Ocyptamus (Orphnabaccha) pteronis } \\
\text { (Fluke, 1942) }\end{array}$} & Tungurahua & $\begin{array}{l}\text { Volcán } \\
\text { Tungurahua, } \\
\text { Minza Chica } \\
(3200) \\
\end{array}$ & Fluke 1942: 5 \\
\hline & Bolívar & Hda. Talahua & Fluke 1942: 5 \\
\hline & Ecuador & & Fluke 1950a: 145 \\
\hline \multirow{4}{*}{$\begin{array}{l}\text { Ocyptamus (Orphnabaccha) trabis } \\
\text { (Fluke, 1942) }\end{array}$} & Tungurahua & \begin{tabular}{|l} 
Volcán \\
Tungurahua, \\
Runtun (2900) \\
\end{tabular} & Fluke 1942: 6 \\
\hline & Imbabura & Cuicocha (3200) & Fluke 1942: 6 \\
\hline & Pichincha & $\begin{array}{l}\text { Páramo de } \\
\text { Pasachoa }\end{array}$ & Fluke 1942: 6 \\
\hline & Ecuador & & Fluke 1950a: 145 \\
\hline $\begin{array}{l}\text { Ocyptamus (Orphnabaccha) virga } \\
\text { (Fluke, 1942) }\end{array}$ & Imbabura & Cuicocha (3200) & Fluke 1942: 7 \\
\hline $\begin{array}{l}\text { Ocyptamus (Orphnabaccha) } \\
\text { volcanus (Fluke, 1942) }\end{array}$ & Pastaza & $\begin{array}{l}\text { Santa Inés, Río } \\
\text { Pastaza (1200) }\end{array}$ & Fluke 1942: 7 \\
\hline $\begin{array}{l}\text { Ocyptamus (Pipunculosyrphus) } \\
\text { scintillans (Hull, 1943) }\end{array}$ & Guayas & Morro (1500) & Hull 1943e: 136 \\
\hline $\begin{array}{l}\text { Ocyptamus (Styxia) eblis } \\
\text { (Hull, 1943) }\end{array}$ & Bolívar & Hda. Talahua & Hull 1943g: 66 \\
\hline Ocyptamus sp. & Guayas & $\begin{array}{l}\text { Guayaquil, San } \\
\text { Eduardo }\end{array}$ & Campos 1960: 24 \\
\hline Ornidia major Curran, 1930 & Ecuador & & $\begin{array}{l}\text { Thompson et al. 1976: 69; Thompson 1991: } \\
255\end{array}$ \\
\hline \multirow{17}{*}{ Ornidia obesa (Fabricius, 1775) } & Galápagos & & Peck 1996 \\
\hline & Galápagos & Isabela & $\begin{array}{l}\text { Peck et al. 1998: 228; Causton et al. 2006: } \\
\text { 135; Sinclair 2015; Sinclair et al. 2016: } 84\end{array}$ \\
\hline & Galápagos & San Cristóbal & $\begin{array}{l}\text { Peck et al. 1998: 228; Causton et al. 2006: } \\
\text { 135; Sinclair 2015; Sinclair et al. 2016: } 84\end{array}$ \\
\hline & Galápagos & Santa Cruz & Sinclair 2015 \\
\hline & Ecuador & & $\begin{array}{l}\text { Thompson et al. 1976: 69; Thompson 1991: } \\
257\end{array}$ \\
\hline & Guayas & Guayaquil & Campos 1960: 26 \\
\hline & Guayas* & El Salado & Campos 1960: 26 \\
\hline & Guayas & San Eduardo & Campos 1960: 26 \\
\hline & Guayas & Durán & Campos 1960: 26 \\
\hline & Guayas & Naranjito & Campos 1960: 26 \\
\hline & Guayas & San Rafael & Campos 1960: 26 \\
\hline & Guayas & Barraganetal & Campos 1960: 26 \\
\hline & Guayas & Bucay & Campos 1960: 26 \\
\hline & Guayas & Posorja & Campos 1960: 26 \\
\hline & Guayas & Playas del Morro & Campos 1960: 26 \\
\hline & Guayas & Naranjal & Campos 1960: 26 \\
\hline & $\begin{array}{l}\text { Zamora } \\
\text { Chinchipe }\end{array}$ & Valle del Zamora & Campos 1960: 26 \\
\hline
\end{tabular}




\begin{tabular}{|c|c|c|c|}
\hline Species & Province & $\begin{array}{c}\text { Locality } \\
\text { (Altitude masl) } \\
\end{array}$ & References for Ecuador \\
\hline \multirow[b]{2}{*}{ Ornidia obesa (Fabricius, 1775) } & Loja & Loja & Campos 1960: 26 \\
\hline & Esmeraldas & $\begin{array}{l}\text { Telembí, Río } \\
\text { Cayapas }\end{array}$ & Campos 1960: 27 \\
\hline Palpada aemula (Williston, 1891) & Ecuador & & $\begin{array}{l}\text { Montoya et al. 2012: supporting information, } \\
\text { page 5; Montoya et al. 2016: } 498\end{array}$ \\
\hline \multirow{5}{*}{$\begin{array}{l}\text { Palpada albifrons } \\
\text { (Wiedemann, 1830) }\end{array}$} & Galápagos & Santa Cruz & Sinclair 2015 \\
\hline & Galápagos & Floreana & Sinclair et al. 2016: 81 \\
\hline & Galápagos & Isabela & Sinclair et al. 2016: 81 \\
\hline & Galápagos & Marchena & Sinclair et al. 2016: 82 \\
\hline & Guayas & San Eduardo & Campos 1960: 28 (as Eristalis albiceps) \\
\hline Palpada atrimana (Loew, 1866) & Ecuador & & Montoya et al. 2016: 496 \\
\hline \multirow[b]{2}{*}{ Palpada conica (Fabricius, 1805) } & Napo & Tena & Morales and Marinoni 2009: 320 \\
\hline & $\begin{array}{l}\text { Zamora } \\
\text { Chinchipe }\end{array}$ & & Morales and Marinoni 2009: 320 \\
\hline Palpada cosmia (Schiner, 1868) & Ecuador & & Thompson et al. 1976: 104 \\
\hline \multirow{3}{*}{ Palpada erratica (Curran, 1930) } & Ecuador & & Thompson et al. 1976: 105 \\
\hline & Azuay & & Morales and Marinoni 2009: 332 \\
\hline & Sucumbíos & & Morales and Marinoni 2009: 332 \\
\hline \multirow{3}{*}{$\begin{array}{l}\text { Palpada fasciata } \\
\text { (Wiedemann, 1819) }\end{array}$} & Ecuador & & Thompson et al. 1976: 105 \\
\hline & Guayas & Guayaquil & Campos 1960: 28 \\
\hline & Guayas & San Eduardo & Campos 1960: 28 \\
\hline Palpada funerea (Rondani, 1851) & Ecuador & Río Napo & Rondani 1851: 357 \\
\hline \multirow{2}{*}{$\begin{array}{l}\text { Palpada furcata } \\
\text { Wiedemann, 1819) }\end{array}$} & Pichincha & Quito & Macquart 1855: 110 (as Eristalis quitensis) \\
\hline & Ecuador & & Thompson et al. 1976: 106 \\
\hline $\begin{array}{l}\text { Palpada geniculata } \\
\text { (Fabricius, 1805) }\end{array}$ & Guayas & Guayaquil & Campos 1960: 28 (as Eristalis obsoletus) \\
\hline Palpada macula (Sack, 1941) & Ecuador & & Thompson et al. 1976: 106 \\
\hline $\begin{array}{l}\text { Palpada mexicana } \\
\text { (Macquart, 1847) }\end{array}$ & Ecuador & & $\begin{array}{l}\text { Thompson et al. 1976: } 110 \text { (as Palpada } \\
\text { testaceicornis) }\end{array}$ \\
\hline \multirow{4}{*}{ Palpada monticola (Röder, 1892) } & Carchi & $\begin{array}{l}10 \mathrm{~km} \text { SW Tulcan } \\
(2900)\end{array}$ & Thompson 1997: 232 (as Palpada eristaloides) \\
\hline & Carchi & Troya $(2950)$ & Thompson 1997: 232 (as Palpada eristaloides) \\
\hline & Azuay & $\begin{array}{l}\text { Cerro Tinajillas } \\
(3100)\end{array}$ & Thompson 1997: 232 (as Palpada eristaloides) \\
\hline & Napo & $\begin{array}{l}0^{\circ} 22^{\prime} \mathrm{S} 78^{\circ} 8^{\prime} \mathrm{W} \\
(3500)\end{array}$ & Thompson 1997: 232 (as Palpada eristaloides) \\
\hline \multirow{2}{*}{ Palpada pusilla (Macquart, 1842) } & Ecuador & & Thompson et al. 1976: 108 \\
\hline & Guayas & Durán & Campos 1960: 28 (as Eristalis tricolor) \\
\hline Palpada pusio (Wiedemann, 1830) & Ecuador & & Thompson et al. 1976: 108 \\
\hline Palpada ruficeps (Macquart, 1842) & Ecuador & & Thompson et al. 1976: 108 \\
\hline $\begin{array}{l}\text { Palpada rufiventris } \\
\text { (Macquart, 1846) }\end{array}$ & Ecuador & & Thompson et al. 1976: 108 \\
\hline \multirow{9}{*}{$\begin{array}{l}\text { Palpada scutellaris } \\
\text { (Fabricius, 1805) }\end{array}$} & Napo & & Morales and Marinoni 2009: 344 \\
\hline & Pastaza & & Morales and Marinoni 2009: 344 \\
\hline & Guayas & Guayaquil & Campos 1960: 28 \\
\hline & Guayas* & El Salado & Campos 1960: 28 \\
\hline & Guayas & San Eduardo & Campos 1960: 28 \\
\hline & Guayas & Durán & Campos 1960: 28 \\
\hline & Guayas & Yaguachi & Campos 1960: 28 \\
\hline & Guayas & Naranjito & Campos 1960: 28 \\
\hline & Guayas & San Rafael & Campos 1960: 28 \\
\hline
\end{tabular}




\begin{tabular}{|c|c|c|c|}
\hline Species & Province & $\begin{array}{c}\text { Locality } \\
\text { (Altitude masl) } \\
\end{array}$ & References for Ecuador \\
\hline \multirow{3}{*}{$\begin{array}{l}\text { Palpada scutellaris } \\
\text { (Fabricius, 1805) }\end{array}$} & Guayas & Bucay & Campos 1960: 28 \\
\hline & Guayas & Posorja & Campos 1960: 28 \\
\hline & Guayas & $\begin{array}{l}\text { Isla Puná, Puerto } \\
\text { Grande }\end{array}$ & Campos 1960: 28 \\
\hline \multirow{4}{*}{$\begin{array}{l}\text { Palpada suprarufa } \\
\text { Thompson, } 1999\end{array}$} & Imbabura & $\begin{array}{l}\text { S Otavalo (3100- } \\
3300)\end{array}$ & Thompson 1999: 345 \\
\hline & Napo & Papallacta $(2900)$ & Thompson 1999: 345 \\
\hline & Pichincha & 28 miles S Quito & Thompson 1999: 345 \\
\hline & Cañar & Pimo (3200) & Thompson 1999: 345 \\
\hline Palpada urotaenia (Curran, 1930) & Ecuador & & Thompson et al. 1976: 110 \\
\hline \multirow{6}{*}{$\begin{array}{l}\text { Palpada vinetorum } \\
\text { (Fabricius, 1799) }\end{array}$} & Galápagos & Española & Sinclair et al. 2016: 82 \\
\hline & Galápagos & San Cristóbal & Sinclair et al. 2016: 82 \\
\hline & Galápagos & Santa Cruz & $\begin{array}{l}\text { Linsley 1977: 39; Sinclair 2015; Sinclair et al. } \\
\text { 2016: } 82\end{array}$ \\
\hline & Ecuador & & Thompson et al. 1976: 110 \\
\hline & Guayas & Guayaquil & Campos 1960: 28 \\
\hline & Guayas & San Eduardo & Campos 1960: 28 \\
\hline \multirow[t]{2}{*}{$\begin{array}{l}\text { Pelecinobaccha adspersa } \\
\text { (Fabricius, 1805) }\end{array}$} & Napo & $\begin{array}{l}\text { Jatun Sacha Biol. } \\
\text { Res. } 6 \text { km E } \\
\text { Misahuali }(450)\end{array}$ & Miranda et al. 2014: 18 \\
\hline & Ecuador & & Thompson et al. 1976: 12 \\
\hline \multirow[t]{2}{*}{$\begin{array}{l}\text { Pelecinobaccha andrettae } \\
\text { Miranda, } 2014\end{array}$} & Napo & $\begin{array}{l}\text { Jatun Sacha Biol. } \\
\text { Res. } 6 \text { km E } \\
\text { Misahuali }(450)\end{array}$ & Miranda et al. 2014: 22 \\
\hline & Pastaza & Pompeya, Napo R. & Miranda et al. 2014: 24 \\
\hline $\begin{array}{l}\text { Pelecinobaccha avispas } \\
\text { Miranda, } 2014\end{array}$ & Napo & $\begin{array}{l}\text { Coca, Napo R. } \\
(250)\end{array}$ & Miranda et al. 2014: 26 \\
\hline $\begin{array}{l}\text { Pelecinobaccha brevipennis } \\
\text { (Schiner, 1868) }\end{array}$ & Napo & $\begin{array}{l}\text { Coca, Napo R. } \\
(250)\end{array}$ & Miranda et al. 2014: 30 \\
\hline $\begin{array}{l}\text { Pelecinobaccha clarapex } \\
\text { (Wiedemann, 1830) }\end{array}$ & Pichincha & $\begin{array}{l}\text { Río Palenque } \\
\text { Station }(250)\end{array}$ & Miranda et al. 2014: 33 \\
\hline $\begin{array}{l}\text { Pelecinobaccha dracula } \\
\text { (Hull, 1943) }\end{array}$ & El Oro & Piñas (1200) & $\begin{array}{l}\text { Hull 1943j: } 215 \text { (as Baccha nerissa); Hull 1949: } \\
162 \text { (as Baccha nerissa) }\end{array}$ \\
\hline Pelecinobaccha ida (Curran, 1941) & Napo & $7 \mathrm{~km} \mathrm{~S} \mathrm{Baeza}$ & Miranda et al. 2014: 49 \\
\hline $\begin{array}{l}\text { Pelecinobaccha ovipositoria } \\
\text { (Hull, 1943) }\end{array}$ & Napo & $\begin{array}{l}\text { Jatun Sacha Biol. } \\
\text { Res. } 6 \text { km E } \\
\text { Misahuali }(450)\end{array}$ & Miranda et al. 2014: 62 \\
\hline \multirow{2}{*}{$\begin{array}{l}\text { Pelecinobaccha pilipes } \\
\text { (Schiner, 1868) }\end{array}$} & Sucumbíos & Limoncocha (250) & Miranda et al. 2014: 67 \\
\hline & Napo & $\begin{array}{l}\text { Coca, Napo R. } \\
(250)\end{array}$ & Miranda et al. 2014: 67 \\
\hline \multirow{7}{*}{$\begin{array}{l}\text { Pelecinobaccha transatlantica } \\
\text { (Schiner, 1868) }\end{array}$} & Napo & $\begin{array}{l}\text { Lago Agrio, } 41 \\
\mathrm{~km} \mathrm{~W}\end{array}$ & Miranda et al. 2014: 78 \\
\hline & Orellana & $\begin{array}{l}\text { Yasuni Research } \\
\text { Stn. (250) }\end{array}$ & Miranda et al. 2014: 78 \\
\hline & Pastaza & Santa Clara & Miranda et al. 2014: 78 \\
\hline & Sucumbíos & Limoncocha (250) & Miranda et al. 2014: 78 \\
\hline & $\begin{array}{l}\text { Zamora } \\
\text { Chinchipe }\end{array}$ & Cumbaratza $(700)$ & Miranda et al. 2014: 78 \\
\hline & Napo & $\begin{array}{l}\text { Puerto Misahuallí } \\
(350)\end{array}$ & Miranda et al. 2014: 78 \\
\hline & Pastaza & Pompeya, Napo R. & Miranda et al. 2014: 78 \\
\hline Peradon aureus (Hull, 1944) & Napo & $\begin{array}{l}\text { Jatun Yacu, Río } \\
\text { Naxo, Watershed } \\
(700)\end{array}$ & Hull 1944a: 36 \\
\hline
\end{tabular}




\begin{tabular}{|c|c|c|c|}
\hline Species & Province & $\begin{array}{c}\text { Locality } \\
\text { (Altitude masl) }\end{array}$ & References for Ecuador \\
\hline \multirow{2}{*}{$\begin{array}{l}\text { Platycheirus (Carposcalis) } \\
\text { chalconotus (Philippi, 1865) }\end{array}$} & Chimborazo & Ríobamba (2700) & Fluke 1945: 16 \\
\hline & Azuay & Cuenca $(2500)$ & Fluke 1945: 16 \\
\hline \multirow{8}{*}{$\begin{array}{l}\text { Platycheirus (Carposcalis) } \\
\text { ecuadoriensis (Fluke, 1945) }\end{array}$} & Imbabura & Cuicocha (3200) & Fluke 1945: 16 \\
\hline & Azuay & Cuenca (2500) & Fluke 1945: 16 \\
\hline & Bolívar & $\begin{array}{l}\text { Hda. Talahua } \\
(3100)\end{array}$ & Fluke 1945: 16 \\
\hline & Chimborazo & Ríobamba (2700) & Fluke 1945: 16 \\
\hline & Chimborazo & Ríobamba (2800) & Fluke 1945: 16 \\
\hline & Pichincha & $\begin{array}{l}\text { Uyumbicho } \\
(2650)\end{array}$ & Fluke 1945: 16 \\
\hline & Pichincha & $\begin{array}{l}\text { Chillo Valley, Hda. } \\
\text { Teno (2500) }\end{array}$ & Fluke 1945: 16 \\
\hline & Ecuador & & Fluke 1958: 265 \\
\hline \multirow{2}{*}{$\begin{array}{l}\text { Platycheirus (Carposcalis) } \\
\text { inflatifrons (Fluke, 1945) }\end{array}$} & Bolívar & $\begin{array}{l}\text { Hda. Talahua } \\
(3100)\end{array}$ & Fluke 1945: 21 \\
\hline & Ecuador & & Fluke 1958: 265 \\
\hline $\begin{array}{l}\text { Platycheirus (Carposcalis) } \\
\text { punctulatus (Wulp, 1888) }\end{array}$ & Ecuador & $(2100-3300)$ & Fluke 1945: 15 \\
\hline $\begin{array}{l}\text { Platycheirus (Carposcalis) cf. } \\
\text { saltanus (Enderlein, 1938) }\end{array}$ & Ecuador & $(4200)$ & Fluke 1945: 15 \\
\hline $\begin{array}{l}\text { Platycheirus (Carposcalis) scutigera } \\
\text { (Fluke, 1945) }\end{array}$ & Pichincha & $\begin{array}{l}\text { Uyumbicho } \\
(2700)\end{array}$ & Fluke 1945: 20 \\
\hline \multirow{5}{*}{$\begin{array}{l}\text { Platycheirus (Carposcalis) stegnus } \\
\text { (Say, 1829) }\end{array}$} & Santa Elena & La Rinconada & Campos 1960: 24 \\
\hline & Chimborazo & Alausí & Campos 1960: 24 \\
\hline & Carchi & El Ángel & Campos 1960: 24 \\
\hline & Pichincha & Casitagua & Campos 1960: 24 \\
\hline & Carchi & Tulcán & Campos 1960: 24 \\
\hline \multirow{15}{*}{$\begin{array}{l}\text { Pseudodoros (Dioprosopa) clavatus } \\
\text { (Fabricius, 1794) }\end{array}$} & Galápagos & Baltra & Sinclair et al. 2016: 89 \\
\hline & Galápagos & Española & Kassebeer 2000: 83; Sinclair et al. 2016: 89 \\
\hline & Galápagos & Genovesa & Kassebeer 2000: 83; Sinclair et al. 2016: 89 \\
\hline & Galápagos & Floreana & $\begin{array}{l}\text { Smith 1877: } 84 \text { (as Syrphus albomaculatus); } \\
\text { Coquillett 1901: 374; Linsley and Usinger } \\
\text { 1966: 168; Linsley 1977: 39; Kassebeer 2000: } \\
\text { 83; Sinclair 2015; Sinclair et al. 2016: } 89\end{array}$ \\
\hline & Galápagos & Isabela & $\begin{array}{l}\text { Curran 1934: 154; Linsley and Usinger 1966: } \\
\text { 168; Linsley 1977: 39; Kassebeer 2000: 83; } \\
\text { Sinclair 2015; Sinclair et al. 2016: } 89\end{array}$ \\
\hline & Galápagos & Pinta & Sinclair et al. 2016: 89 \\
\hline & Galápagos & Marchena & $\begin{array}{l}\text { Linsley 1977: 39; Sinclair 2015; Sinclair et al. } \\
\text { 2016: } 89\end{array}$ \\
\hline & Galápagos & San Cristóbal & $\begin{array}{l}\text { Curran 1934: 154; Linsley and Usinger 1966: } \\
\text { 168; Linsley 1977: 39; Sinclair 2015; Sinclair } \\
\text { et al. 2016: } 89\end{array}$ \\
\hline & Galápagos & Rábida & Sinclair et al. 2016: 89 \\
\hline & Galápagos & Santiago & $\begin{array}{l}\text { Coquillett 1901: 374; Linsley and Usinger } \\
\text { 1966: 168; Linsley 1977: 39; Kassebeer 2000: } \\
83\end{array}$ \\
\hline & Galápagos & Santa Fé & Sinclair et al. 2016: 89 \\
\hline & Galápagos & Bartolomé & Kassebeer 2000: 83 \\
\hline & Galápagos & Seymour Norte & Johnson 1924: 88 \\
\hline & Galápagos & Santa Cruz & $\begin{array}{l}\text { Linsley 1977: 39; Kassebeer 2000: 83; Sinclair } \\
\text { 2015; Sinclair et al. 2016: } 89\end{array}$ \\
\hline & Galápagos & & Thomson 1869: 548 (as Baccha facialis) \\
\hline
\end{tabular}




\begin{tabular}{|c|c|c|c|}
\hline Species & Province & $\begin{array}{c}\text { Locality } \\
\text { (Altitude masl) } \\
\end{array}$ & References for Ecuador \\
\hline \multirow{4}{*}{$\begin{array}{l}\text { Pseudodoros (Dioprosopa) clavatus } \\
\text { (Fabricius, } 1794 \text { ) }\end{array}$} & Azuay & $\begin{array}{l}32 \mathrm{~km} \text { W Santa } \\
\text { Isabel }(900)\end{array}$ & Kassebeer 2000: 85 \\
\hline & Manabí & $\begin{array}{l}\text { Manta-Jipijapa rd. } \\
(150)\end{array}$ & Kassebeer 2000: 85 \\
\hline & $\begin{array}{l}\text { Zamora } \\
\text { Chinchipe }\end{array}$ & Zamora (1500) & Kassebeer 2000: 85 \\
\hline & \begin{tabular}{|l} 
Zamora \\
Chinchipe
\end{tabular} & $\begin{array}{l}\text { Loja, San Pedro } \\
(1550)\end{array}$ & Kassebeer 2000: 85 \\
\hline \multirow{4}{*}{$\begin{array}{l}\text { Pseudodoros (Dioprosopa) vockerothi } \\
\text { (Kassebeer, 2000) }\end{array}$} & Bolívar & $\begin{array}{l}\text { Chota River, } \\
\text { Carchi }(1800)\end{array}$ & Kassebeer 2000: 76 \\
\hline & Imbabura & $\begin{array}{l}\text { Ibarra, } \\
\text { Yaguarcocha } \\
(2300)\end{array}$ & Kassebeer 2000: 76 \\
\hline & Loja & $\begin{array}{l}\text { S. Pedro-Zaruma } \\
\text { rd Loja }(850- \\
1100)\end{array}$ & Kassebeer 2000: 76 \\
\hline & Imbabura & $\begin{array}{l}\text { Taguando R., NW } \\
\text { Ibarra }(1650- \\
1900)\end{array}$ & Kassebeer 2000: 76 \\
\hline Quichuana aff. quixotea Hull, 1946 & Napo & Limoncocha & Ricarte et al. 2012: 129 \\
\hline \multirow{4}{*}{ Relictanum crassum (Walker, 1852) } & Cotopaxi & Latacunga (330) & Miranda et al. 2014: 91 \\
\hline & Los Ríos & $\begin{array}{l}\text { Río Palenque } \\
(150)\end{array}$ & Miranda et al. 2014: 91 \\
\hline & Napo & $\begin{array}{l}\text { Puerto Misahuallí } \\
(350)\end{array}$ & Miranda et al. 2014: 91 \\
\hline & Sucumbíos & Limoncocha (250) & Miranda et al. 2014: 91 \\
\hline $\begin{array}{l}\text { Relictanum johnsoni } \\
\text { (Curran, 1934) }\end{array}$ & Napo & \begin{tabular}{|l|} 
Coca, Napo R. \\
$(250)$
\end{tabular} & Miranda et al. 2014: 93 \\
\hline $\begin{array}{l}\text { Rhingia (Rhingia) longirostris } \\
\text { Fluke, } 1943\end{array}$ & Bolívar & $\begin{array}{l}\text { Hda. Talahua } \\
(3100)\end{array}$ & Fluke 1943: 431 \\
\hline $\begin{array}{l}\text { Rhingia (Rhingia) nigra } \\
\text { Macquart, } 1846\end{array}$ & Ecuador & & Montoya et al. 2016: 506 \\
\hline Rhinoprosopa lucifer (Hull, 1943) & El Oro & Piñas (1600) & Hull 1943j: 216 \\
\hline Rhinoprosopa nasuta (Bigot, 1884) & Carchi & R. Chota (2000) & Mengual 2015: 16 \\
\hline $\begin{array}{l}\text { Rhopalosyrphus ecuadoriensis } \\
\text { Reemer, } 2013\end{array}$ & Orellana & $\begin{array}{l}\text { Yasuni Research } \\
\text { Station }\end{array}$ & Reemer and Ståhls 2013a: 119 \\
\hline $\begin{array}{l}\text { Salpingogaster browni } \\
\text { Curran, } 1941\end{array}$ & Tungurahua & $\begin{array}{l}\text { Volcán } \\
\text { Tungurahua, } \\
\text { Minza Chica } \\
(3200) \\
\end{array}$ & Curran 1941: 286 \\
\hline \multirow{6}{*}{$\begin{array}{l}\text { Scaeva melanostoma } \\
\text { (Macquart, 1842) }\end{array}$} & Azuay & & Thompson et al. 1976: 9 \\
\hline & Pichincha & \begin{tabular}{|l|}
$2 \mathrm{~km}$ W Cayambe \\
$(2300)$
\end{tabular} & Kassebeer 1999: 99 \\
\hline & Carchi & El Ángel (2700) & Kassebeer 1999: 99 \\
\hline & Pichincha & $\begin{array}{l}\text { Valle de Machachi } \\
(2900)\end{array}$ & Kassebeer 1999: 99 \\
\hline & Chimborazo & Riobamba & Campos 1960: 29; Kassebeer 1999: 99 \\
\hline & Chimborazo & env. of Riobamba & Kassebeer 1999: 99 \\
\hline Scaeva occidentalis Shannon, 1927 & Pichincha & $\begin{array}{l}\text { Valle de Machachi } \\
(2900)\end{array}$ & Kassebeer 1999: 101 \\
\hline $\begin{array}{l}\text { Sterphus (Crepidomyia) chloropyga } \\
\text { (Schiner, 1868) }\end{array}$ & Ecuador & & $\begin{array}{l}\text { Schiner 1868: } 366 \text { (type-locality as } \\
\text { "Colombien", referring to Colombia, Ecuador, } \\
\text { or Venezeula); Montoya et al. 2016: } 504\end{array}$ \\
\hline
\end{tabular}




\begin{tabular}{|c|c|c|c|}
\hline Species & Province & $\begin{array}{c}\text { Locality } \\
\text { (Altitude masl) } \\
\end{array}$ & References for Ecuador \\
\hline \multirow{3}{*}{$\begin{array}{l}\text { Sterphus (Crepidomyia) plagiatus } \\
\text { (Wiedemann, 1830) }\end{array}$} & Napo & $\begin{array}{l}\text { Napo River, Coca } \\
(250)\end{array}$ & Thompson 1973: 220 \\
\hline & Napo & Napo River & Thompson 1973: 220 \\
\hline & Pastaza & $\begin{array}{l}\text { Napo River, } \\
\text { Pompeya }\end{array}$ & Thompson 1973: 220 \\
\hline $\begin{array}{l}\text { Sterphus (Telus) telus } \\
\text { Thompson, } 1973\end{array}$ & Azuay & Tarqui (2800) & Thompson 1973: 198 \\
\hline \multirow{2}{*}{$\begin{array}{l}\text { Stipomorpha guianica } \\
\text { (Curran, 1925) }\end{array}$} & $\begin{array}{l}\text { Morona } \\
\text { Santiago }\end{array}$ & $\begin{array}{l}\text { Limón Indanza } \\
(900)\end{array}$ & Reemer 2013: 54 \\
\hline & Ecuador & & Thompson et al. 1976: 62 \\
\hline $\begin{array}{l}\text { Stipomorpha tenuicauda } \\
\text { (Curran, 1925) }\end{array}$ & Napo & $\begin{array}{l}\text { Jatun Sacha Res., } \\
6 \text { km E Misahualli } \\
(450)\end{array}$ & Reemer 2013: 70 \\
\hline Stipomorpha zophera Reemer, 2013 & Napo & Limoncocha & Reemer 2013: 75 \\
\hline $\begin{array}{l}\text { Syrphus aff. lacyorum } \\
\text { Thompson, } 2000\end{array}$ & $\begin{array}{l}\text { Morona } \\
\text { Santiago }\end{array}$ & Río Blanco & Thompson et al. 2000: 39 \\
\hline Syrphus reedi Shannon, 1927 & $\begin{array}{l}\text { Zamora } \\
\text { Chinchipe }\end{array}$ & Valle de Zamora & Campos 1960: 25 \\
\hline \multirow{4}{*}{ Syrphus shorae Fluke, 1950} & Tungurahua & $\begin{array}{l}\text { Bańos }(1500- \\
2100)\end{array}$ & Fluke 1942: 3 (as S. willistoni) \\
\hline & Tungurahua & Juive (1950) & Fluke 1942: 3 (as S. willistoni) \\
\hline & Pichincha & $\begin{array}{l}\text { Hda. San Rafael, } \\
\text { Río San Pedro } \\
(2700)\end{array}$ & Fluke 1942: 3 (as S. willistoni) \\
\hline & Ecuador & & Fluke 1950a: 143 (as S. willistoni) \\
\hline \multirow[t]{2}{*}{ Talahua fervida (Fluke, 1945) } & Bolívar & $\begin{array}{l}\text { Hda. Talahua } \\
(3100)\end{array}$ & Fluke 1945: 23 \\
\hline & Ecuador & & Fluke 1958: 266 \\
\hline \multirow{21}{*}{ Toxomerus anthrax (Schiner, 1868) } & Ecuador & & Thompson et al. 1976: 48; Mengual 2011: 9 \\
\hline & Pastaza & Abitagua Oriente & Gerdes 1974a: 14-15 \\
\hline & Tungurahua & Bańos & Gerdes 1974a: 14-15 \\
\hline & Pastaza & Cerro Obitahua & Gerdes 1974a: 14-15 \\
\hline & Ecuador** & Conquista & Gerdes 1974a: 14-15 \\
\hline & Tungurahua & Naguazo & Gerdes 1974a: 14-15 \\
\hline & Napo & Napo Oriente & Gerdes 1974a: 14-15 \\
\hline & Pastaza & Obitahua Oriente & Gerdes 1974a: 14-15 \\
\hline & $\begin{array}{l}\text { Morona } \\
\text { Santiago } \\
\end{array}$ & Río Blanco & Gerdes 1974a: 14-15 \\
\hline & \begin{tabular}{|l|} 
Morona \\
Santiago \\
\end{tabular} & Río Negro & Gerdes 1974a: 14-15 \\
\hline & Tungurahua & Runtun & Gerdes 1974a: 14-15 \\
\hline & Chimborazo & Sangay Oriente & Gerdes 1974a: 14-15 \\
\hline & Pastaza & Puerto Santana & Gerdes 1974a: 14-15 \\
\hline & Pastaza & Sarayacu & Gerdes 1974a: 14-15 \\
\hline & Pastaza & Sarayacu Oriente & Gerdes 1974a: 14-15 \\
\hline & Pastaza & El Topo & Gerdes 1974a: 14-15 \\
\hline & Pichincha & Chaupi & Gerdes 1974a: 14-15 \\
\hline & Tungurahua & Ulvilla & Gerdes 1974a: 14-15 \\
\hline & Chimborazo & Chilicay & Mengual 2011: appendix 1 \\
\hline & Chimborazo & Huigra & Mengual 2011: appendix 1 \\
\hline & El Oro & Portovelo & Mengual 2011: appendix 1 \\
\hline
\end{tabular}




\begin{tabular}{|c|c|c|c|}
\hline Species & Province & $\begin{array}{c}\text { Locality } \\
\text { (Altitude masl) }\end{array}$ & References for Ecuador \\
\hline \multirow[t]{2}{*}{ Toxomerus antiopa (Hull, 1951) } & Bolívar & $\begin{array}{l}\text { Hda. Talahua } \\
(3100)\end{array}$ & Hull 1951: 5 \\
\hline & Chimborazo & Urbina $(3650)$ & Hull 1951: 5 \\
\hline Toxomerus aquilinus Sack, 1941 & Ecuador & & Metz and Thompson 2001: 233 \\
\hline Toxomerus arcifer (Loew, 1866) & Ecuador & & Thompson et al. 1976: 48 \\
\hline \multirow{21}{*}{ Toxomerus brevifacies (Hull, 1943) } & Tungurahua & $\begin{array}{l}\text { Bańos, Runtun } \\
\text { trail }\end{array}$ & Hull 1943g: 20 \\
\hline & Imbabura & Cuicocha & Hull 1943g: 20 \\
\hline & Pastaza & San Francisco & Hull 1943g: 20 \\
\hline & Tungurahua & Juive & Hull 1943g: 20 \\
\hline & Tungurahua & Baños & Hull 1943g: 20; Gerdes 1974a: 19 \\
\hline & Azuay & Cuenca & Hull 1943g: 20 \\
\hline & Tungurahua & Bańos & Gerdes 1974a: 19; Gerdes 1975: 20 \\
\hline & Pichincha & Chaupi & Gerdes 1974a: 20; Gerdes 1975: 20 \\
\hline & Ecuador** & Conquista & Gerdes 1974a: 20; Gerdes 1975: 20 \\
\hline & Pastaza & Obitagua & Gerdes 1974a: 20; Gerdes 1975: 20 \\
\hline & $\begin{array}{l}\text { Morona } \\
\text { Santiago }\end{array}$ & Río Blanco & Gerdes 1974a: 20; Gerdes 1975: 20 \\
\hline & $\begin{array}{l}\text { Morona } \\
\text { Santiago }\end{array}$ & Río Negro & Gerdes 1974a: 20; Gerdes 1975: 20 \\
\hline & Tungurahua & Runtun & Gerdes 1974a: 20; Gerdes 1975: 20 \\
\hline & Chimborazo & Sangay Oriente & Gerdes 1974a: 20; Gerdes 1975: 20 \\
\hline & Pastaza & Sarayacu & Gerdes 1974a: 20; Gerdes 1975: 20 \\
\hline & Pastaza & Sarayacu Oriente & Gerdes 1974a: 20; Gerdes 1975: 20 \\
\hline & Pastaza & Topo & Gerdes 1974a: 20; Gerdes 1975: 20 \\
\hline & Tungurahua & Ulvilla & Gerdes 1974a: 20; Gerdes 1975: 20 \\
\hline & \begin{tabular}{|l|} 
Pastaza \\
\end{tabular} & Abitagua Oriente & Gerdes 1974a: 20; Gerdes 1975: 20 \\
\hline & \begin{tabular}{|l|} 
Tungurahua \\
\end{tabular} & Naguazo & Gerdes 1974a: 20; Gerdes 1975: 20 \\
\hline & Pastaza & Puerto Santana & Gerdes 1974a: 20; Gerdes 1975: 20 \\
\hline \multirow{3}{*}{ Toxomerus claracuneus (Hull, 1942) } & Pastaza & \begin{tabular}{|l|} 
Río Margaritas, \\
Río Pastaza $(1250)$ \\
\end{tabular} & Hull 1942: 107 \\
\hline & Ecuador** & Conquista & Gerdes 1974a: 22 \\
\hline & Pastaza & Puerto Santana & Gerdes 1974a: 22 \\
\hline \multirow{8}{*}{ Toxomerus crockeri (Curran, 1934) } & Galápagos & Floreana & $\begin{array}{l}\text { Curran 1934: 155; Linsley and Usinger 1966: } \\
\text { 168; Linsley 1977: 39; Sinclair and Peck 2002; } \\
\text { Sinclair 2015; Sinclair et al. 2016: } 91\end{array}$ \\
\hline & Galápagos & Isabela & $\begin{array}{l}\text { Curran 1934: 155; Linsley and Usinger 1966: } \\
\text { 168; Linsley 1977: 39; Peck 1994; Sinclair and } \\
\text { Peck 2002; Boada 2005: 80; Sinclair 2015; } \\
\text { Sinclair et al. 2016: } 91\end{array}$ \\
\hline & Galápagos & Pinta & Sinclair and Peck 2002 \\
\hline & Galápagos & San Cristóbal & $\begin{array}{l}\text { Curran 1934: 155; Linsley and Usinger 1966: } \\
\text { 168; Linsley 1977: 39; Sinclair and Peck 2002; } \\
\text { Sinclair et al. 2016: } 91\end{array}$ \\
\hline & Galápagos & Santiago & $\begin{array}{l}\text { Coquillett 1901: } 374 \text { (as Mesogramma } \\
\text { duplicata); Curran 1934: 155; Linsley and } \\
\text { Usinger 1966: 168; Linsley 1977: 39; Sinclair } \\
\text { and Peck 2002 }\end{array}$ \\
\hline & Galápagos & Española & Sinclair et al. 2016: 91 \\
\hline & Galápagos & Pinta & Sinclair et al. 2016: 91 \\
\hline & Galápagos & Santa Cruz & $\begin{array}{l}\text { Curran 1934: 155; Boada 2005: 85; Sinclair } \\
\text { 2015; Sinclair et al. 2016: } 91\end{array}$ \\
\hline
\end{tabular}




\begin{tabular}{|c|c|c|c|}
\hline Species & Province & $\begin{array}{c}\text { Locality } \\
\text { (Altitude masl) } \\
\end{array}$ & References for Ecuador \\
\hline \multirow{4}{*}{ Toxomerus dispar (Fabricius, 1794) } & Tungurahua & Baños & $\begin{array}{l}\text { Hull 1943f: } 26 \text { (as Mesogramma basilaris var. } \\
\text { bifida); Gerdes 1974a: } 17\end{array}$ \\
\hline & Napo & Napo Oriente & Gerdes 1974a: 17 (as Toxomerus basilaris) \\
\hline & $\begin{array}{l}\text { Morona } \\
\text { Santiago }\end{array}$ & Río Blanco & Gerdes 1974a: 17 (as Toxomerus basilaris) \\
\hline & Ecuador & & Mengual 2011: 13 \\
\hline \multirow{3}{*}{$\begin{array}{l}\text { Toxomerus duplicatus } \\
\text { (Wiedemann, 1830) }\end{array}$} & Pichincha & Pichincha & Hull 1943f: 18 (as Mesogramma arcturus) \\
\hline & Pichincha & Tío Loma & Campos 1960: 25 \\
\hline & Napo & Napo Oriente & Gerdes 1974a: 23 \\
\hline \multirow{16}{*}{ Toxomerus ecuadoreus (Hull, 1943) } & Azuay & Cuenca (2500) & Hull 1943g: 20 \\
\hline & Tungurahua & Baños (2200) & Hull 1943g: 20 \\
\hline & Pichincha & Pichincha (2500) & Hull 1943g: 20 \\
\hline & Pichincha & $\begin{array}{l}\text { Hda. San Rafael, } \\
\text { Río San Pedro }\end{array}$ & Hull 1943g: 20; Gerdes 1974a: 26 \\
\hline & Pichincha & Uyumbicho & Hull 1943g: 20; Gerdes 1974a: 26 \\
\hline & Tungurahua & $\begin{array}{l}\text { Bańos, Río Pablo } \\
(2200)\end{array}$ & Hull 1943g: 20 \\
\hline & Tungurahua & Baños, Runtun & Hull 1943g: 20 \\
\hline & Chimborazo & Ríobamba (2700) & Hull 1943g: 20 \\
\hline & Pichincha & Aloag & Gerdes 1974a: 26; Gerdes 1975: 22 \\
\hline & Tungurahua & Baños & Gerdes 1975: 22 \\
\hline & Pastaza & Obitagua & Gerdes 1974a: 26; Gerdes 1975: 22 \\
\hline & $\begin{array}{l}\text { Morona } \\
\text { Santiago } \\
\end{array}$ & Río Blanco & Gerdes 1974a: 26; Gerdes 1975: 22 \\
\hline & Pichincha & Chaupi & Gerdes 1974a: 26 \\
\hline & \begin{tabular}{|l|} 
Tungurahua \\
\end{tabular} & Ulvilla & Gerdes 1974a: 26 \\
\hline & $\begin{array}{l}\text { Morona } \\
\text { Santiago } \\
\end{array}$ & Río Negro & Gerdes 1974a: 26; Gerdes 1975: 22 \\
\hline & Tungurahua & Runtun & Gerdes 1974a: 26; Gerdes 1975: 22 \\
\hline \multirow{13}{*}{ Toxomerus flaviplurus (Hall, 1927) } & Pastaza & Cerro Obitahua & Gerdes 1974a: 31 \\
\hline & Napo & Napo Oriente & Gerdes 1974a: 31 \\
\hline & Pastaza & Puyo Oriente & Gerdes 1974a: 31 \\
\hline & \begin{tabular}{|l|} 
Chimborazo \\
\end{tabular} & Sangay Oriente & Gerdes 1974a: 31 \\
\hline & Pastaza & $\begin{array}{l}1.5 \mathrm{~km} \mathrm{~S} \text { Puyo, } \\
\text { Río Pido Grande }\end{array}$ & Mengual 2011: appendix 1 \\
\hline & Tungurahua & $\begin{array}{l}32 \text { km E Bańos } \\
(1560)\end{array}$ & Mengual 2011: appendix 1 \\
\hline & Napo & Tena & Mengual 2011: appendix 1 \\
\hline & Napo & Santa Cecilia & Mengual 2011: appendix 1 \\
\hline & Napo & $\begin{array}{l}60 \mathrm{~km} \mathrm{~W} \\
\text { LagoAgRío }\end{array}$ & Mengual 2011: appendix 1 \\
\hline & Napo & Limoncocha & Mengual 2011: appendix 1 \\
\hline & $\begin{array}{l}\text { Zamora } \\
\text { Chinchipe } \\
\end{array}$ & Zumbi & Mengual 2011: appendix 1 \\
\hline & $\begin{array}{l}\text { Zamora } \\
\text { Chinchipe }\end{array}$ & Cumbaratza & Mengual 2011: appendix 1 \\
\hline & $\begin{array}{l}\text { Zamora } \\
\text { Chinchipe }\end{array}$ & Yantzaza & Mengual 2011: appendix 1 \\
\hline \multirow{2}{*}{ Toxomerus floralis (Fabricius, 1789) } & Ecuador & & Thompson and Thompson 2007: 324 \\
\hline & Napo & Napo Oriente & Gerdes 1974a: 35 \\
\hline
\end{tabular}




\begin{tabular}{|c|c|c|c|}
\hline Species & Province & $\begin{array}{c}\text { Locality } \\
\text { (Altitude masl) } \\
\end{array}$ & References for Ecuador \\
\hline \multirow{9}{*}{$\begin{array}{l}\text { Toxomerus hieroglyphicus } \\
\text { (Schiner, 1868) }\end{array}$} & Tungurahua & Baños & Gerdes 1974a: 37; Mengual 2011: appendix 1 \\
\hline & Ecuador & & Thompson et al. 1976: 51; Mengual 2011: 16 \\
\hline & Pastaza & Cerro Obitahua & Gerdes 1974a: 37 \\
\hline & Pastaza & Obitahua Oriente & Gerdes 1974a: 37 \\
\hline & $\begin{array}{l}\text { Morona } \\
\text { Santiago } \\
\end{array}$ & Río Blanco & Gerdes 1974a: 37 \\
\hline & Tungurahua & Runtun & Gerdes 1974a: 38 \\
\hline & Chimborazo & Sangay Oriente & Gerdes 1974a: 38 \\
\hline & Pastaza & Abitagua Oriente & Gerdes 1974a: 38 \\
\hline & Ecuador** & Conquista & Gerdes 1974a: 38 \\
\hline \multirow{2}{*}{ Toxomerus idalius (Hull, 1951) } & Pastaza & Puyo (1000) & $\begin{array}{l}\text { Hull 1951: 12; Hull 1951: } 13 \text { (as Mesogramma } \\
\text { idalia leda) }\end{array}$ \\
\hline & Pastaza & $\begin{array}{l}\text { Río Pastaza, San } \\
\text { Francisco }(1200)\end{array}$ & $\begin{array}{l}\text { Hull 1951: } 13 \text { (as Mesogramma idalia leda); } \\
\text { Hull 1951: } 18 \text { (as Mesogramma eurydice) }\end{array}$ \\
\hline \multirow{7}{*}{ Toxomerus insignis (Schiner, 1868) } & Ecuador & & $\begin{array}{l}\text { Thompson et al. 1976: } 50 \text { (as T. elongatus); } \\
\text { Metz and Thompson 2001: } 235\end{array}$ \\
\hline & Tungurahua & Baños & Gerdes 1974a: 29 (as Toxomerus elongatus) \\
\hline & Napo & Napo Oriente & Gerdes 1974a: 29 (as Toxomerus elongatus) \\
\hline & Tungurahua & Ulvilla & Gerdes 1974a: 29 (as Toxomerus elongatus) \\
\hline & Pastaza & Abitagua & Gerdes 1974a: 29 (as Toxomerus elongatus) \\
\hline & Pastaza & Sarayacu & Gerdes 1974a: 29 (as Toxomerus elongatus) \\
\hline & Pastaza & Abitagua & Gerdes 1974a: 29 (as Toxomerus elongatus) \\
\hline \multirow{5}{*}{ Toxomerus lacrymosus (Bigot, 1884) } & Napo & Napo Oriente & Gerdes 1974a: 40 \\
\hline & Pastaza & Obitahua Oriente & Gerdes 1974a: 40 \\
\hline & \begin{tabular}{l|} 
Nariño \\
{$[\text { Colombia }]^{* *}$}
\end{tabular} & Piedrancha & Gerdes 1974a: 40 \\
\hline & Chimborazo & Sanqay Oriente & Gerdes 1974a: 40 \\
\hline & Pastaza & Sarayacu & Gerdes 1974a: 40 \\
\hline Toxomerus laenas (Walker, 1852) & Ecuador & & Thompson et al. 1976: 53 (as T. nitidiventris) \\
\hline Toxomerus marginatus (Say, 1823) & $\begin{array}{l}\text { Cañar- } \\
\text { Chimborazo }\end{array}$ & Quinua-Loma & Campos 1960: 25 \\
\hline \multirow{8}{*}{$\begin{array}{l}\text { Toxomerus minutus } \\
\text { (Wiedemann, 1830) }\end{array}$} & Pichincha & Casitagua & Campos 1960: 26 \\
\hline & Carchi & El Vínculo & Campos 1960: 26 \\
\hline & Azuay & Borma & Campos 1960: 26 \\
\hline & Santa Elena & La Rinconada & Campos 1960: 26 \\
\hline & $\begin{array}{l}\text { Cańar- } \\
\text { Chimborazo } \\
\end{array}$ & Quinua-Loma & Campos 1960: 26 \\
\hline & $\begin{array}{l}\text { Santo } \\
\text { Domingo de } \\
\text { los Tsáchilas } \\
\end{array}$ & $\begin{array}{l}\text { Santo Domingo de } \\
\text { los Colorados }\end{array}$ & Campos 1960: 26 \\
\hline & Carchi & Tulcán & Campos 1960: 26 \\
\hline & Loja & Loja & Campos 1960: 26 \\
\hline \multirow{8}{*}{ Toxomerus nasutus Sack, 1941} & Pichincha & $\begin{array}{l}\text { Uyumbicho } \\
(2700)\end{array}$ & Hull 1951: 8 (as Mesogramma ultima) \\
\hline & Tungurahua & Baños (2500) & Hull 1943c: 36 (as Mesogramma sylpha) \\
\hline & Tungurahua & Baños (1800) & Hull 1943c: 36 (as Mesogramma sylpha) \\
\hline & Tungurahua & Baños & Gerdes 1975: 14 \\
\hline & Pichincha & Chaupi & Gerdes 1974a: 42; Gerdes 1975: 14 \\
\hline & Ecuador** & Conquista & Gerdes 1974a: 42; Gerdes 1975: 14 \\
\hline & Tungurahua & Naguazo & Gerdes 1974a: 42; Gerdes 1975: 14 \\
\hline & Napo & Napo Oriente & Gerdes 1974a: 42; Gerdes 1975: 14 \\
\hline
\end{tabular}




\begin{tabular}{|c|c|c|c|}
\hline Species & Province & $\begin{array}{c}\text { Locality } \\
\text { (Altitude masl) } \\
\end{array}$ & References for Ecuador \\
\hline \multirow{15}{*}{ Toxomerus nasutus Sack, 1941} & Pastaza & Obitagua & Gerdes 1974a: 42; Gerdes 1975: 14 \\
\hline & Pastaza & Obitahua & Gerdes 1974a: 42; Gerdes 1975: 14 \\
\hline & Pastaza & Abitagua Oriente & Gerdes 1974a: 42 \\
\hline & Pastaza & Cerro Obitahua & Gerdes 1974a: 42 \\
\hline & Manabí* & San José & Gerdes 1974a: 42 \\
\hline & Tungurahua & El Topo & Gerdes 1974a: 43 \\
\hline & $\begin{array}{l}\text { Morona } \\
\text { Santiago }\end{array}$ & Río Blanco & Gerdes 1974a: 42; Gerdes 1975: 14 \\
\hline & $\begin{array}{l}\text { Morona } \\
\text { Santiago } \\
\end{array}$ & Río Negro & Gerdes 1974a: 42; Gerdes 1975: 14 \\
\hline & Tungurahua & Runtun & Gerdes 1974a: 42; Gerdes 1975: 14 \\
\hline & Tungurahua* & El Salado & Gerdes 1974a: 42; Gerdes 1975: 14 \\
\hline & Chimborazo & Sangay & Gerdes 1974a: 43; Gerdes 1975: 14 \\
\hline & Pastaza & Puerto Santana & Gerdes 1974a: 43; Gerdes 1975: 14 \\
\hline & Pastaza & Sarayacu & Gerdes 1974a: 43; Gerdes 1975: 14 \\
\hline & Pastaza & Sarayacu Oriente & Gerdes 1974a: 43; Gerdes 1975: 14 \\
\hline & Pichincha* & Yunguilla & Gerdes 1974a: 44; Gerdes 1975: 14 \\
\hline Toxomerus norma (Hull, 1941) & Ecuador & & $\begin{array}{l}\text { Thompson et al. 1976: } 52 \text { (as T. mulio); Metz } \\
\text { and Thompson 2001: } 239 \text { (as T. mulio) }\end{array}$ \\
\hline \multirow{9}{*}{ Toxomerus nymphalius (Hull, 1942) } & Pastaza & $\begin{array}{l}\text { Río Margaritas } \\
(1250)\end{array}$ & Hull 1942: 106 \\
\hline & $\begin{array}{l}\text { Morona } \\
\text { Santiago } \\
\end{array}$ & $\begin{array}{l}\text { Sucúa, Río Blanco } \\
(950)\end{array}$ & Hull 1942: 106 \\
\hline & Pastaza & Puyo & Hull 1942: 106 \\
\hline & Pastaza & Río Mapeto & Hull 1942: 106 \\
\hline & Pastaza & Cerro Obitahua & Gerdes 1974a: 46 \\
\hline & Pastaza & Obitahua Oriente & Gerdes 1974a: 46 \\
\hline & Chimborazo & Sangay Oriente & Gerdes 1974a: 46 \\
\hline & Pastaza & Sasayacu Oriente & Gerdes 1974a: 46 \\
\hline & Pichincha* & Yunguilla & Gerdes 1974a: 46 \\
\hline Toxomerus parvulus (Loew, 1866) & Ecuador & & Thompson et al. 1976: 55 (as T. slossonae) \\
\hline Toxomerus pichinchae Gerdes, 1974 & Pichincha & Aloag $(2600)$ & Gerdes 1974b: 280 \\
\hline \multirow{3}{*}{ Toxomerus pictus (Macquart, 1842) } & Pastaza & Cerro Obitahua & Gerdes 1974a: 48 \\
\hline & Chimborazo & Sangay Oriente & Gerdes 1974a: 48 \\
\hline & Napo & Napo Oriente & Gerdes 1974a: 49 \\
\hline Toxomerus picudus Mengual, 2011 & Orellana & $\begin{array}{l}\text { Estación Tiputini } \\
(227)\end{array}$ & Mengual 2011: 21 \\
\hline \multirow{10}{*}{ Toxomerus politus (Say, 1823) } & Galápagos & Floreana & Sinclair 2015 \\
\hline & Galápagos & Isabela & Sinclair 2015 \\
\hline & Galápagos & Santa Cruz & Sinclair et al. 2016: 93 \\
\hline & Galápagos & San Cristóbal & Sinclair 2015 \\
\hline & Galápagos & Santiago & Sinclair et al. 2016: 93 \\
\hline & Tungurahua & Bańos & Gerdes 1974a: 51 \\
\hline & Napo & Napo Oriente & Gerdes 1974a: 51 \\
\hline & $\begin{array}{l}\text { Nariño } \\
{[\text { Colombia }]^{* *}}\end{array}$ & Piedrancha & Gerdes 1974a: 51 \\
\hline & Pastaza & Sarayacu & Gerdes 1974a: 51 \\
\hline & Ecuador & & $\begin{array}{l}\text { Thompson et al. 1976: 53; Metz and } \\
\text { Thompson 2001: } 241\end{array}$ \\
\hline $\begin{array}{l}\text { Toxomerus porticola } \\
\text { (Thomson, 1869) }\end{array}$ & Ecuador & & Thompson et al. 1976: 54 \\
\hline
\end{tabular}




\begin{tabular}{|c|c|c|c|}
\hline Species & Province & $\begin{array}{c}\text { Locality } \\
\text { (Altitude masl) }\end{array}$ & References for Ecuador \\
\hline \multirow{12}{*}{$\begin{array}{l}\text { Toxomerus productus } \\
\text { (Curran, 1930) }\end{array}$} & $\begin{array}{l}\text { Morona } \\
\text { Santiago }\end{array}$ & $\begin{array}{l}\text { Macas, Río Upano } \\
(1000)\end{array}$ & Hull 1951: 10 (as Mesogramma cyrilla) \\
\hline & Ecuador & & Curran 1930: 5 \\
\hline & Napo & Napo Oriente & Gerdes 1974a: 53; Gerdes 1975: 16 \\
\hline & Pastaza & Obitahua & Gerdes 1974a: 53; Gerdes 1975: 16 \\
\hline & Chimborazo & Sangay & Gerdes 1974a: 53; Gerdes 1975: 16 \\
\hline & Pastaza & Sarayacu & Gerdes 1974a: 53; Gerdes 1975: 16 \\
\hline & Pastaza & Sarayacu & Gerdes 1974a: 54; Gerdes 1975: 16 \\
\hline & Tungurahua & Baños & Gerdes 1974a: 54; Gerdes 1975: 16 \\
\hline & Pastaza & Obitagua & Gerdes 1974a: 54; Gerdes 1975: 16 \\
\hline & $\begin{array}{l}\text { Morona } \\
\text { Santiago }\end{array}$ & Río Negro & Gerdes 1974a: 54; Gerdes 1975: 16 \\
\hline & Tungurahua & Runtun & Gerdes 1974a: 54; Gerdes 1975: 16 \\
\hline & Pastaza & Sarayacu Oriente & Gerdes 1974a: 54; Gerdes 1975: 16 \\
\hline $\begin{array}{l}\text { Toxomerus rombicus (Giglio- } \\
\text { Tos, 1892) }\end{array}$ & Azuay & Cuenca & Campos 1960: 25 \\
\hline \multirow{9}{*}{$\begin{array}{l}\text { Toxomerus saphiridiceps } \\
\text { (Bigot, 1884) }\end{array}$} & Ecuador & & $\begin{array}{l}\text { Thompson et al. 1976: } 50 \text { (as T. flavus), 54; } \\
\text { Metz and Thompson 2001: } 246\end{array}$ \\
\hline & Tungurahua & Bańos & Gerdes 1974a: 33 (as Toxomerus flavus) \\
\hline & Ecuador** & Conquista & Gerdes 1974a: 34 (as Toxomerus flavus) \\
\hline & $\begin{array}{l}\text { Morona } \\
\text { Santiago }\end{array}$ & Río Blanco & Gerdes 1974a: 34 (as Toxomerus flavus) \\
\hline & Manabí & San José & Gerdes 1974a: 34 (as Toxomerus flavus) \\
\hline & $\begin{array}{l}\text { Narińo } \\
{[\text { Colombia }]^{* *}}\end{array}$ & Piedrancha & Gerdes 1974a: 34 (as Toxomerus flavus) \\
\hline & Tungurahua & Runtun & Gerdes 1974a: 34 (as Toxomerus flavus) \\
\hline & Pastaza & Sarayacu & Gerdes 1974a: 34 (as Toxomerus flavus) \\
\hline & Los Ríos & Soledad & Gerdes 1974a: 34 (as Toxomerus flavus) \\
\hline Toxomerus sp. & Galápagos & Santa Cruz & Boada 2005: 86 \\
\hline \multirow{3}{*}{ Toxomerus sp. } & Guayas & San Eduardo & Campos 1960: 26 \\
\hline & Guayas & Guayaquil & Campos 1960: 26 \\
\hline & Guayas & Durán & Campos 1960: 26 \\
\hline \multirow{4}{*}{ Toxomerus steatogaster (Hull, 1941) } & $\begin{array}{l}\text { Morona } \\
\text { Santiago }\end{array}$ & $\begin{array}{l}\text { Sucúa, Río Blanco } \\
\text { and Río Upano } \\
(950)\end{array}$ & Hull 1943f: 21 (as Mesogramma steatornis) \\
\hline & Pastaza & Puyo (1000) & Hull 1943f: 21 (as Mesogramma steatornis) \\
\hline & Napo & Napo Oriente & Gerdes 1974a: 55 \\
\hline & Ecuador & & Thompson et al. 1976: 55 \\
\hline \multirow{6}{*}{ Toxomerus sylvaticus (Hull, 1943) } & Tungurahua & Baños & Hull 1943c: 35; Gerdes 1974a: 57 \\
\hline & Pastaza & Cerro Obitahua & Gerdes 1974a: 57 \\
\hline & Pastaza & Obitahua Oriente & Gerdes 1974a: 57 \\
\hline & $\begin{array}{l}\text { Morona } \\
\text { Santiago }\end{array}$ & Río Blanco & Gerdes 1974a: 57 \\
\hline & Chimborazo & Sanqay Oriente & Gerdes 1974a: 57 \\
\hline & Pichincha & Chaupi & Gerdes 1974a: 57 \\
\hline $\begin{array}{l}\text { Toxomerus tibicen } \\
\text { (Wiedemann, 1830) }\end{array}$ & Guayas & $\begin{array}{l}\text { Guayaquil, San } \\
\text { Eduardo }\end{array}$ & Campos 1960: 25 \\
\hline Toxomerus tubularius (Hull, 1942) & Tungurahua & Baños (2000) & Hull 1942: 104 \\
\hline
\end{tabular}




\begin{tabular}{|c|c|c|c|}
\hline Species & Province & $\begin{array}{c}\text { Locality } \\
\text { (Altitude masl) }\end{array}$ & References for Ecuador \\
\hline $\begin{array}{l}\text { Toxomerus virgulatus } \\
\text { (Macquart, 1850) }\end{array}$ & Ecuador & & Thompson et al. 1976: 49 (as T. confusus) \\
\hline Toxomerus watsoni (Curran, 1930) & Ecuador & & Thompson et al. 1976: 56 \\
\hline \multirow{2}{*}{$\begin{array}{l}\text { Tuberculanostoma antennatum } \\
\text { Fluke, } 1943\end{array}$} & Bolívar & Talahua (3100) & Fluke 1943: 426 \\
\hline & Ecuador & & Fluke 1958: 266 \\
\hline \multirow{3}{*}{$\begin{array}{l}\text { Tuberculanostoma browni } \\
\text { Fluke, } 1943\end{array}$} & Chimborazo & Urbina (3650) & Fluke 1943: 429 \\
\hline & Bolívar & $\begin{array}{l}\text { Hda. Talahua } \\
(3100)\end{array}$ & Fluke 1943: 430 \\
\hline & Bolívar & $\begin{array}{l}\text { Cumbre de Tililac } \\
(4200)\end{array}$ & Fluke 1943: 430 \\
\hline \multirow[t]{2}{*}{$\begin{array}{l}\text { Tuberculanostoma cilium } \\
\text { Fluke, } 1943\end{array}$} & Tungurahua & \begin{tabular}{|l|} 
Volcán \\
Tungurahua, \\
Minza Chica \\
$(3200)$ \\
\end{tabular} & Fluke 1943: 428 \\
\hline & Bolívar & $\begin{array}{l}\text { Hda. Talahua } \\
(3100)\end{array}$ & Fluke 1943: 428 \\
\hline $\begin{array}{l}\text { Tuberculanostoma pectinis } \\
\text { Fluke, } 1943\end{array}$ & Bolívar & \begin{tabular}{|l|}
$\begin{array}{l}\text { Hda. Talahua } \\
(3100)\end{array}$ \\
\end{tabular} & Fluke 1943: 430 \\
\hline Ubristes ictericus Reemer, 2013 & Sucumbíos & Sach Lodge (270) & Reemer 2013: 80 \\
\hline \multirow[b]{2}{*}{$\begin{array}{l}\text { Xanthandrus (Xanthandrus) } \\
\text { palliatus (Fluke, 1945) }\end{array}$} & Bolívar & $\begin{array}{l}\text { Hda. Talahua } \\
(3100)\end{array}$ & Fluke 1945: 22 \\
\hline & Tungurahua & \begin{tabular}{|l} 
Volcán \\
Tungurahua, \\
Minza Chica \\
$(3200)$ \\
\end{tabular} & Fluke 1945: 22 \\
\hline
\end{tabular}

Some original locality names were difficult to place in the current administrative divisions of Ecuador. The Río Pastaza (= Pastaza river) runs through two Ecuadorian provinces, i.e. Pastaza and Morona Santiago, and we used Pastaza province for this locality. On the other hand, Quinua Loma is a locality situated between two provinces, Cañar and Chimborazo, and we listed both provinces in Table 1.

Most of the uncertainties on geographical localities come from Gerdes (1974a). For instance, Gerdes (1974a) named three localities as different ones, i.e. Obitagua, Obitahua, and Abitagua, although we believe that they might refer to the same area. There is a single locality named Abitagua in Ecuador, but instead of assuming all being the same locality, we left the three names in Table 1. We are not sure if the locality San José (Gerdes 1974a) is the one currently situated in Manabí, and there are two localities named El Salado in Guayas (between 0 and 200 masl) and in Tungurahua (circa 2,000 masl). We listed El Salado in Guayas for the records of Campos (1960), as most of the records in that work were from Guayas, but we used Tungurahua for El Salado of Gerdes (1974a, 1975) for the record of Toxomerus nasutus Sack, 1941 because other records for this species are close to or over 2,000 masl. We had a similar problem with Yunguilla, a locality also found in two different provinces (Azuay and Pichincha), and we used Pichincha in 
this case because Gerdes had studied material from Pichincha but not from Azuay. All these records are marked with an asterisk $\left({ }^{*}\right)$ in the Province column of Table 1.

The locality Piedrancha belongs to Colombia (Nariño department), but it was left in Table 1 because Gerdes (1974a) listed it as Ecuador. Finally, we were not able to locate Conquista in Ecuador. These records are marked with two asterisks $\left.{ }^{(*)}\right)$ in the Province column of Table 1.

For the elaboration of Tables 1 and 2, the most recent Syrphidae classification has been used (Mengual et al. 2008, 2009, Thompson 2012, 2013, Reemer and Ståhls 2013a, Miranda et al. 2014, 2016, Mengual 2015). Flower fly species recorded in Ecuador are listed in Table 1 in alphabetical order. Genera with the highest number of species were Toxomerus (38), Ocyptamus (22) and Palpada (21) (Table 2).

Four unidentified species are listed as such (Microdon sp., Ocyptamus sp. and two Toxomerus sp.), and three species are affinis to known species, Dasysyrphus aff. lotus, Syrphus aff. lacyorum and Quichuana aff. quixotea. Ricarte et al. (2012) reviewed the taxonomy of the genus Quichuana Knab, 1913 and mentioned one Quichuana species recorded for Ecuador (Ricarte et al. 2012: 129, Figure 84). The identity of this species was not stated by Ricarte et al. (2012), but personal communication with A. Ricarte revealed that it is Quichuana aff. quixotea (Hull 1946). Four specimens from Ecuador labelled as Q. quixoted are known to be deposited in the USNM collection. However, they show some morphological differences with the holotype that prevented Ricarte et al. (2012) to ascertain their identity (Antonio Ricarte, pers. comm.).

There was some ambiguity with Peradon oligonax (Hull, 1944) to either include it or not in the checklist. Peradon oligonax was described from Pto. America, Río Putumayo (Hull 1944c). Thompson et al. (1976: 66) indicated the type-locality as part of Ecuador, but Hull (1944c: 36) listed it as Brazil. Putumayo River forms part of Colombia's border with Ecuador, as well as most of the frontier with Peru, and it ends as a tributary of the Amazon River in Brazil, but there it is known as Içá. Rasmussen (2016) gave details of the Cornell University expedition to South America (collectors of the type material) and he provided evidences that the expedition never went to Ecuador and the expedition was near Javary island (Santo Antônio do Içá) in the dates when the type material was collected. Thus, the type-locality is in Brazil and not in Ecuador, as indicated by Thompson et al. (1976).

Another uncertain taxon was Priomerus gagathinus Bigot, 1887, originally described from Loja. Thompson et al (1976) declared the type of this taxon as lost and did not recognize the species. Thompson (2015) indicated that the name Priomerus was preoccupied and its species currently belong to four different genera. He did not recognize either the species gagathinus Bigot. Thus, we did not list this species in Table 1.

In the literature, we found two doubtful species records, probably due to a misidentification. Sphaerophoria (Sphaerophoria) sulphuripes (Thomson, 1869) is a Nearctic species found along the west coast of the United States and Canada (Knutson 1973). Thompson et al. (1976: 38) listed one specimen identified as S. sulphuripes (with no details about the responsible of this identification) in The Natural History Museum 
Table 2. Number of genera and species registered in Ecuador.

\begin{tabular}{|c|c|}
\hline Genus & Number of species in Ecuador \\
\hline Alipumilio Shannon, 1927 & 1 \\
\hline Allograpta Osten Sacken, 1875 & 9 \\
\hline Argentinomyia Lynch Arribalzaga, 1891 & 10 \\
\hline Claraplumula Shannon, 1927 & 1 \\
\hline Copestylum Macquart, 1846 & 19 \\
\hline Dasysyrphus Enderlein, 1938 & 1 \\
\hline Dolichogyna Macquart, 1842 & 2 \\
\hline Eosalpingogaster Hull, 1949 & 1 \\
\hline Eristalis Latreille, 1804 & 1 \\
\hline Eupeodes Osten Sacken, 1877 & 1 \\
\hline Fazia Shannon, 1927 & 12 \\
\hline Hypselosyrphus Hull, 1937 & 1 \\
\hline Leucopodella Hull, 1949 & 4 \\
\hline Lycastrirhyncha Bigot, 1859 & 1 \\
\hline Mallota Meigen, 1822 & 3 \\
\hline Meromacrus Rondani, 1848 & 3 \\
\hline Meropidia Hippa \& Thompson, 1983 & 1 \\
\hline Microdon Meigen, 1803 & 3 \\
\hline Mixogaster Macquart, 1842 & 1 \\
\hline Ocyptamus Macquart, 1834 & 22 \\
\hline Ornidia Lepeletier \& Serville, 1828 & 2 \\
\hline Palpada Macquart, 1834 & 21 \\
\hline Pelecinobaccha Shannon, 1927 & 10 \\
\hline Peradon Reemer, 2013 & 1 \\
\hline Platycheirus Lepeletier \& Serville, 1828 & 7 \\
\hline Pseudodoros Becker, 1903 & 2 \\
\hline Quichuana Knab, 1913 & 1 \\
\hline Relictanum Miranda, 2014 & 2 \\
\hline Rhingia Scopoli, 1763 & 2 \\
\hline Rhinoprosopa Hull, 1942 & 2 \\
\hline Rhopalosyrphus Giglio-Tos, 1891 & 1 \\
\hline Salpingogaster Schiner, 1868 & 1 \\
\hline Scaeva Fabricius, 1805 & 2 \\
\hline Sterphus Philippi, 1865 & 3 \\
\hline Stipomorpha Hull, 1945 & 3 \\
\hline Syrphus Fabricius, 1775 & 3 \\
\hline Talahua Fluke, 1945 & 1 \\
\hline Toxomerus Macquart, 1855 & 38 \\
\hline Tuberculanostoma Fluke, 1943 & 4 \\
\hline Ubristes Walker, 1852 & 1 \\
\hline Xanthandrus Verrall, 1901 & 1 \\
\hline
\end{tabular}


(BMNH, London, U.K.) from Ecuador with a question mark. This specimen might be mislabeled or it could be an Allograpta specimen, most likely a female, somehow similar to $S$. sulphiripes. We believe that $S$. sulphuripes does not occur in Ecuador and it was not included in Table 1. The other taxon that was misidentified is Eristalis (Eoseristalis) pertinax (Scopoli, 1763), identified by Campos (1960). This species ranges from Fennoscandia south to Iberia and the Mediterranean, and from Ireland through much of Europe into European parts of Russia and Turkey; apparently it is not known beyond the Urals (Speight 2016). We do believe that the record might be an Eristalis species, but not E. pertinax as it does not occur in the Neotropics. Thus, this record is not listed in Table 1.

Three species are not listed due to the uncertainty of their taxonomic identity. Syrphus excavatus (Rondani 1851: 359) and Syrphus fasciventris (Rondani 1851:360), both described from Río Napo, are not included because the type material was not studied and the generic name is probably incorrect. The third species not included is Xanthandrus sp. (Curran 1934: 155; from Pinta Island, Galapagos). Sinclair et al. (2016) could not find the material studied by Curran to confirm if the specimen from Galapagos is truly Xanthandrus or Argentinomyia agonis (Walker 1849).

\section{Discussion}

Montoya et al. (2012) recorded 128 species of 40 different genera for Ecuador, indicating that Ecuador shares a high number of species with Brazil (29 species), Colombia (50) and Peru (29). The present work raises those numbers considerably, up to 201 identified species of 51 genera and subgenera. Based on previous studies, the Ecuadorian diversity of flower flies is comparable to the one from Peru (195 spp., 75 genera; Montoya et al. 2012), Costa Rica (228 species, 41 genera; Montoya et al. 2012) or Suriname (183 species, 36 genera; Reemer 2016). It is important to emphasize that Ecuador is one of the smallest countries in the Neotropics and South America, but it has one of the highest diversity densities for the Neotropics with ca. 7.2 species per 10.000 $\mathrm{km} 2$. This diversity density makes Ecuador the third top country after Costa Rica and Suriname, the two most explored and well-studied faunae in the Neotropics. It must also be pointed out that the present work is based only on records from the literature, and authors are sure that the flower fly diversity in Ecuador is higher.

This study confirms the argument of Montoya et al. (2012) when stating that "The understanding of the distribution and composition of Syrphidae in the Neotropical Region remains far from complete". Since Thompson et al. (1976) there have been mostly taxonomic contributions on the Neotropical flower flies, but little faunistic studies have been published. Thompson (1999) provided a key to the Neotropical genera of Syrphidae, including a glossary of taxonomic terms and the description of a few new species, and Thompson (2006) compiled all the taxonomic knowledge of Neotropical flower flies up to that date, but those cannot be considered faunistic studies. In the Systema Dipterorum, Thompson (2013) had some distributional range notes for each species, but the fauna of the Neotropical countries has not been studied more thoroughly yet. The 
syrphid fauna of three Neotropical countries have been recently revised: a catalogue for Colombia (Montoya 2016, see also Gutierrez et al. 2005), another online catalogue for Brazil (Morales and Marinoni 2017), and an extensive taxonomic study of the flower flies of Suriname (Reemer 2010, 2014, 2016). In addition, Thompson et al. (2010) gave a very comprehensive synopsis of the Central American Syrphidae.

Thompson et al. (2010) stated that ca. 1,800 flower fly species are described from the Neotropical Region, but other authors argue that this may be only half of the actual number of species (Reemer 2016). Thus, Ecuadorian syrphid fauna comprises roughly $11.2 \%$ of the described Neotropical species. Emulating the arguments of Reemer (2016), the syrphid fauna of Ecuador might be two to four times larger, up to 900 species, if we compare the known species of other taxa in this country with the total number of species in the Neotropical Region. Cárdenas et al. (2009) estimated that Ecuador has 16.3\% of the Neotropical species of the family Tabanidae (Diptera). Mittermeier et al. (2005) calculated that the bird species present in Ecuador are ca. $47 \%$ of the total number of species in the Neotropics. With an estimate of 4,000 species of butterflies (Salazar and Donoso 2014, M. Espeland pers. comm.), Ecuador probably hosts half of the Neotropical diversity of this order. In other words, considering these numbers and the fact that Syrphidae is underexplored in Ecuador (Amorim 2009), we are far from having a good estimate of the total number of flower fly species for Ecuador.

We think that the inventory and study of the Syrphidae fauna are essential not only to describe new species from Ecuador, but also to help in the selection of areas to protect, based on species richness, and to improve the management of conservation areas in this country. Salazar and Donoso (2014) mentioned that the taxonomic complexity, the lack of experts for some groups, the high species richness, and the endemicity of many invertebrates in Ecuador make the study of its invertebrate fauna a major challenge in science. Moreover, Ecuador has two biodiversity hotspot regions: Tropical Andes and Tumbes-Chocó-Magdalena (Myers et al. 2000, Mittermeier et al. 2004). These regions are heavily threatened and need urgent conservation efforts. In such cases, faunistic studies should have priority to understand the biological diversity of those hotspots. Furthermore, the poor knowledge of the relationships between flower flies and their prey, as well as the unknown associations with host plants, make the study of this group essential 1) to improve our understanding about their roles in the ecosystem performance and organic matter decomposition, 2) to evaluate the biological richness of Ecuador in order to establish new management and control protocols over its natural resources, and 3) to revise the quarantine and international trade policies for preventing potential pest species dispersal and creating new banned species list.

\section{Acknowledgments}

We thank the Alexander Koenig Gesellschaft (AKG) for funding support for field equipment to start the preliminary studies on Syrphidae in Ecuador. We are grateful 
to the Departamento de Ciencias Biológicas (UTPL), Carlos Iván Espinosa, Augusta Cueva, and Carlos Naranjo for funding support for the organization of a Syrphidae Workshop. We also thank Ximena Cueva and Ángel Romero for their help with literature and laboratory work. XM thanks the library staff, especially the Digitization department, of the Western Illinois University for providing a copy of the PhD thesis of Charles Frederick Gerdes. We thank Gil F. Miranda and Mírian N. Morales for their comments and suggestions, which greatly improved our manuscript.

\section{References}

Adler PH, Foottit RG (2009) Introduction. In: Foottit RG, Adler PH (Eds) Insect Biodiversity: Science and Society. Blackwell Publishing, Oxford, 1-6. http://doi. org/10.1002/9781444308211.ch1

Amorim DS (2009) Neotropical Diptera diversity: richness, patterns, and perspectives. In: Pape T, Bickel D, Meier R (Eds) Diptera Diversity: Status, Challenges and Tools. Brill, Leiden, 71-97. https://doi.org/10.1163/ej.9789004148970.I-459.17

Amorós-Jiménez R, Pineda A, Fereres A, Marcos-García MA (2014) Feeding preferences of the aphidophagous hoverfly Sphaerophoria rueppellii affect the performance of its offspring. BioControl 59: 427-435. https://doi.org/10.1007/s10526-014-9577-8

Barragán A, Dangles O, Cárdenas R, Onore G (2009) The History of Entomology in Ecuador. Annals of the Entomological Society of America 45(4): 410-423. http://doi.org/10.1080 /00379271.2009.10697626

Bergh JC, Short BD (2008) Ecological and life-history notes on syrphid predators of woolly apple aphid in Virginia, with emphasis on Heringia calcarata. BioControl 53: 773-786. https://doi.org/10.1007/s10526-007-9114-0

Bigot JM (1887) Note sur divers Diptères de Yeso (Japon) et de Loja (Equateur); note suivie de la description de nouvelles espèces. Annales de la Société Entomologique de France Serie 6/7(Bul.): 80-86.

Blatch S, Thompson FC, Zumbado MA (2003) The Mesoamerican Meromacrus flower flies (Diptera: Syrphidae). Studia Dipterologica 10: 13-36.

Boada R (2005) Insects associated with endangered plants in the Galápagos Islands, Ecuador. Entomotropica 20(2): 77-88. http://hdl.handle.net/1807/37799

Bodner F, Brehm G, Homeier J, Strutzenberger P, Fiedler K (2010) Caterpillars and host plant records for 59 species of Geometridae (Lepidoptera) from a montane rainforest in southern Ecuador. Journal of Insect Science 10(1): 67. https://doi.org/10.1673/031.010.6701

Bollino M, Onore G (2001) Mariposas del Ecuador. Vol. 10 A. Papilionidae. Pontificia Universidad Católica del Ecuador, Quito, $171 \mathrm{pp}$.

Brehm G (2005) A revision of the Acrotomodes clota Druce, 1900 species-group (Lepidoptera: Geometridae, Ennominae). Entomologische Zeitschrift 115: 75-80. http://www.gunnarbrehm.de/tl_files/DATA/pdf-Dateien/Brehm_2005_EZ_Acrotomodes2.pdf

Brehm G, Homeier J, Fiedler K, Kottke I, Illig J, Nöske NM, Werner FA, Breckle SW (2008) Mountain rain forests in southern Ecuador as a hotspot of biodiversity - limited knowl- 
edge and diverging patterns. In: Beck E, Bendix J, Kottke I, Makeschin F, Mosandl Rm (Eds) Gradients in a tropical mountain ecosystem of Ecuador. Ecological Studies 198. Springer, 15-24. https://doi.org/10.1007/978-3-540-73526-7_2

Brown BV (2009) Introduction. In: Brown BV, Borkent A, Cumming JM, Wood DM, Woodley NE, Zumbado MA (Eds) Manual of Central American Diptera, Vol. 1. NRC CNRC Research Press, Ottawa, 1-7.

Bugg RL, Colfer RG, Chaney WE, Smith HA, Cannon J (2008) Flower flies (Syrphidae) and other biological control agents for aphids in vegetable crops. UC ANR Publication 8285. http://anrcatalog.ucanr.edu/pdf/8285.pdf

Campos F (1960) Las Moscas (Brachycera) del Ecuador. Revista Ecuatoriana de Higiene y Medicina Tropical 17: 1-66.

Cárdenas RE, Buestán J, Dangles O (2009) Diversity and distribution models of horse flies (Diptera: Tabanidae) from Ecuador. Annales de la Société Entomologique de France 45(4): 511-528. https://doi.org/10.1080/00379271.2009.10697633

Carpio C, Donoso DA, Ramón G, Dangles O (2009) Short term response of dung beetle communities to disturbance by road construction in the Ecuadorian Amazon. Annales de la Société Entomologique de France 45(4): 455-469. https://doi.org/10.1080/00379271.2 009.10697629

Carvajal V, Villamarín S, Ortega AM (2011) Escarabajos del Ecuador. Principales Géneros. Instituto de Ciencias Biológicas. Escuela Politécnica Nacional. Serie Entomología Nro 1.

Causton CE, Peck SB, Sinclair BJ, Roque-Albelo L, Hodgson CJ, Landry B (2006) Alien insects: threats and implications for conservation of Galápagos Islands. Annals of the Entomological Society of America 99(1): 121-143. http://doi.org/10.1603/00138746(2006)099[0121:AITAIF]2.0.CO;2

Celi J, Terneus E, Torres J, Ortega M (2004) Dung beetles (Coleoptera: Scarabaeinae) diversity in an altitudinal gradient in the Cutucú range, Morona Santiago, Ecuadorian Amazon. Lyonia 7(2): 37-52. http://www.lyonia.org/downloadPDF.php?pdfID=2.247.1

Coquillett DW (1901) Papers from, the Hopkins Galapagos Expedition, 1898-1899. II. Entomological Results (2): Dipteria [sic]. Proceedings of the Washington Academy of Sciences 3: 371-379. http://doi.org/10.5962/bhl.part.26342

Courtney GW, Pape T, Skevington JH, Sinclair BJ (2009) Biodiversity of Diptera. In: Foottit RG, Adler PH (Eds) Insect Biodiversity: Science and Society. Blackwell Publishing, Oxford, 185-222. https://doi.org/10.1002/9781444308211.ch9

Curran CH (1930) New Diptera belonging to the genus Mesogramma Loew (Syrphidae). American Museum Novitates 405: 1-14. http://hdl.handle.net/2246/4363

Curran CH (1934) The Templeton Crocker Expedition of the California Academy of Sciences, 1932. No. 13. Diptera. Proceedings of the California Academy of Sciences 21: 147-172.

Curran CH (1939) Synopsis of the American species of Volucella (Syrphidae; Diptera). Part II. Descriptions of New Species. American Museum Novitates 1028: 1-17. http://hdl.handle. net/2246/2219

Curran CH (1940) Some new Neotropical Syrphidae (Diptera). American Museum Novitates 1086: 1-14. http://hdl.handle.net/2246/2238 
Curran CH (1941) New American Syrphidae. Bulletin of the American Museum of Natural History 78: 243-304.

Curran CH (1953) Notes and description of some Mydaidae and Syrphidae. American Museum Novitates 16145: 1-15. http://hdl.handle.net/2246/2413

Dangles O, Barragan A, Cardenas RE, Onore G, Keil C (2009) Entomology in Ecuador: Recent developments and future challenges. Annales de la Société Entomologique de France 45(4): 424-436. http://doi.org/10.1080/00379271.2009.10697627

Domínguez D, Marín-Armijos D, Ruiz C (2015) Structure of dung beetle communities in an altitudinal gradient of Neotropical dry forest. Neotropical entomology 44(1): 40-46. https://doi.org/10.1007/s13744-014-0261-6

Dumbardon-Martial E (2016) Pollen feeding in the larva of Toxomerus pulchellus (Diptera, Syrphidae). Bulletin de la Société Entomologique de France 121(4): 413-420. http://doi. org/10.1080/00222930802610576

Eckberg JO, Peterson JA, Borsh CP, Kaser JM, Johnson GA, Luhman JC, Wyse DL, Heimpel GE (2015) Field abundance and performance of hoverflies (Diptera: Syrphidae) on soybean aphid. Annals of the Entomological Society of America 108(1): 26-34. http://doi. org/10.1093/aesa/sau009

Fiedler K, Brehm G, Hilt N, Süssenbach D, Onore G, Bartsch D, Racheli L, Häuser CL (2007) Lepidoptera: Arctiidae, Geometridae, Hedylidae, Pyraloidea, Sphingidae, Saturniidae, and Uraniidae (Moths) Checklists - Reserva Biológica San Francisco (Prov. Zamora-Chinchipe, S. Ecuador). In: Liede-Schumann S, Breckle SW (Eds) Provisional checklists of flora and fauna of the San Francisco Valley and its surroundings (Reserva Biológica San Francisco, Prov. Zamora-Chinchipe, Southern Ecuador). Ecotropical Monographs No.4, 256 pp.

Fleischmann A, Rivadavia F, Gonella PM, Pérez-Bañón C, Mengual X, Rojo S (2016) Where is my food? Brazilian flower fly steals prey from carnivorous sundews in a newly discovered plant-animal interaction. PLoS ONE 11(5): e0153900. http://doi.org/10.1371/journal. pone. 0153900

Fluke CL (1937) New South American Syrphidae (Diptera). American Museum Novitates 941: 1-14. http://hdl.handle.net/2246/2182

Fluke CL (1942) Revision of the Neotropical Syrphini related to Syrphus. American Museum Novitates 1201: 1-24. http://hdl.handle.net/2246/2279

Fluke CL (1943) A new genus and new species of Syrphidae (Diptera) from Ecuador. Annals of the Entomological Society of America 36(3): 425-431. http://doi.org/10.1093/ aesa/36.3.425

Fluke CL (1945) The Melanostomini of the Neotropical Region (Diptera, Syrphidae). American Museum Novitates 1272: 1-29. http://hdl.handle.net/2246/3720

Fluke CL (1950a) The male genitalia of Syrphus, Epistrophe and related genera (Diptera, Syrphidae). Transactions of the Wisconsin Academy of Sciences Arts and Letters 40: 115-148.

Fluke CL (1950b) Some new tropical syrphid flies with notes on others. Acta Zoológica Lilloana 9: 439-454.

Fluke CL (1951a) The genus "Dolichogyna” (Diptera, Syrphidae). Acta Zoológica Lilloana 12: $465-478$. 
Fluke CL (1951b) Syrphid flies related to Volucella scutellata Macquart. American Museum

Novitates 1503: 1-33. http://hdl.handle.net/2246/2367

Fluke CL (1958) A study of the male genitalia of the Melanostomini (Diptera-Syrphidae).

Transactions of the Wisconsin Academy of Sciences Arts and Letters 46: 261-279.

Gerdes CF (1974a) Toxomerus (Diptera: Syrphidae) of Ecuador. PhD thesis, Western Illinois University, $135 \mathrm{pp}$.

Gerdes CF (1974b) A new species of Ecuadorean Toxomerus (Diptera: Syrphidae). Entomological News 85: 279-283.

Gerdes CF (1975) Notes on types of Toxomerus (Diptera: Syrphidae). Entomological News 86: $13-22$.

Greco CF (1998) Aphidophagous syrphids (Diptera, Syrphidae): quick identification of preimaginal stages and list of natural enemies of the most commonly found species in cereals and pastures in the province of Buenos Aires (Argentina). Acta Entomológica Chilena 22: 7-11.

Gutierrez C, Carrejo NS, Ruiz C (2005) Listado de los géneros de Syrphidae (Diptera: Syrphoidea) de Colombia. Biota Colombiana 6(2): 173-180. http://icn.unal.edu.co/publicaciones/art/206/6-N2/syrphidae.pdf

Hilt N, Fiedler K (2005) Diversity and composition of Arctiidae moth ensembles along a successional gradient in the Ecuadorian Andes. Diversity and Distributions 11(5): 387-398. https://doi.org/10.1111/j.1366-9516.2005.00167.x

Hippa H, Thompson FC (1983) Meropidia, a new genus of flower flies (Diptera: Syrphidae) from South America. Papéis Avulsos de Zoologia 35(9): 109-115. https://repository. si.edu/handle/10088/18911

Hull FM (1942) New species of Syrphidae from the Neotropical region. Psyche 49: 84-107. https://doi.org/10.1155/1942/62725

Hull FM (1943a) Two new species of Baccha (Syrphidae). Proceedings of the Entomological Society of Washington 45: 50-51.

Hull FM (1943b) Some flies of the genus Volucella in the British Museum (Natural History) Annals And Magazine of Natural History 10: 18-40. https://doi.org/10.1080/03745481 .1943 .9727992

Hull FM (1943c) Some new American Syrphid flies (Diptera). Entomological News 54: 29-37. Hull FM (1943d) New species of Syrphidae of the genus Baccha and Mesogramma. Entomological News 54: 89-91.

Hull FM (1943e) New species of Baccha and related flies. Entomological News 54: 135-140.

Hull FM (1943f) The genus Mesogramma. Entomologica Americana 23: 1-41.

Hull FM (1943g) The New World species of the genus Baccha. Entomologica Americana 23: 42-99.

Hull FM (1943h) New species of Syrphid flies in the National Museum. Journal of the Washington Academy of Sciences 33: 39-43.

Hull FM (1943i) Some undescribed species of the genus Baccha (Syrphidae). Journal of the Washington Academy of Sciences 33: 72-74.

Hull FM (1943j) New species of the genera Baccha and Rhinoprosopa (Syrphidae). Journal of the Washington Academy of Sciences 33: 214-216. 
Hull FM (1944a) Studies on Syrphid flies in the Museum of Comparative Zoology. Psyche 51: 22-45. https://doi.org/10.1155/1944/61923

Hull FM (1944b) Additional species of the genus Baccha from the New World. Bulletin of the Brooklyn Entomological Society 39: 56-64.

Hull FM (1944c) A study of some Syrphid flies of South America. Revista de Entomologia, Rio de Janeiro 15: 34-54.

Hull FM (1949) The genus Baccha from the New World. Entomologica Americana 21(1947): 89-291.

Hull FM (1950) New South American Syrphid flies. Revista de Entomologica, Rio de Janeiro 21: 225-236.

Hull FM (1951) New species of Mesogramma (Diptera: Syrphidae). American Museum Novitates 1480: $1-22$.

Inouye D, Larson BMH, Ssymank A, Kevan PG (2015) Flies and flowers III: Ecology of foraging and pollination. Journal of Pollination Ecology 16 (16): 115-133.

Johnson CW (1924) Diptera of the Williams Galapagos Expedition. Zoologica 5(8): 85-92.

Jordaens K, Goergen G, Kirk-Spriggs AH, Vokaer A, Backljau T, De Meyer M (2015) A second New World hoverfly, Toxomerus floralis (Fabricius) (Diptera: Syrphidae), recorded from the Old World, with description of larval pollen-feeding ecology. Zootaxa 4044(4): 567-576. http://doi.org/10.11646/zootaxa.4044.4.6

Kassebeer CF (1999) Die neotropischen Arten der Gattung Scaeva Fabricius, 1805 (Diptera, Syrphidae). Dipteron 2(5): 93-108.

Kassebeer CF (2000) Zur Gattung Pseudodoros Becker, 1903 (Diptera, Syrphidae). Dipteron 3(1): 73-92.

Knutson LV (1973) Taxonomic revision of the aphid-killing flies of the genus Sphaerophoria in the Western hemisphere (Syrphidae). Miscellaneous Publications of the Entomological Society of America 9(1): 1-50.

Lardé G (1989) Investigation on some factors affecting larval growth in a coffee-pulp bed. Biological Wastes 30: 11-19. https://doi.org/10.1016/0269-7483(89)90139-0

Linsley EG (1977) Insects of the Galápagos (Supplement). Occasional Papers of the California Academy of Sciences 125: 1-55.

Linsley EG, Usinger RL (1966) Insects of the Galápagos Islands. Proceedings of the California Academy of Sciences, Serie 4(33): 113-196.

Macquart J (1855) Dipteres exotiques nouveaux ou peu connus. 5e supplement. Mémoires de la Société des Sciences, de l'Agriculture et des Arts de Lille 1854: 25-156, 7.

Marinoni L, Thompson FC (2003) Flower flies of southern Brazil (Diptera: Syrphidae). Part I. Introduction and new species. Studia Dipterologica 10(2): 565-578.

Martínez-Falcón AP, Marcos-García MA, Moreno CE, Rotheray GE (2012) A critical role for Copestylum larvae (Diptera, Syrphidae) in the decomposition of cactus forests. Journal of Arid Environments 78: 41-48. http://doi.org/10.1016/j.jaridenv.2011.10.010

Mengual X (2011) Black-tie dress code: two new species of the genus Toxomerus (Diptera, Syrphidae). ZooKeys 140: 1-26. http://doi.org/10.3897/zookeys.140.1930

Mengual X (2015) Revision of the genus Rhinoprosopa (Diptera: Syrphidae). The Canadian Entomologist 147: 1-22. http://doi.org/10.4039/tce.2014.25 
Mengual X, Ruiz C, Rojo S, Ståhls G, Thompson FC (2009) A conspectus of the flower fly genus Allograpta (Diptera: Syrphidae) with description of a new subgenus and species. Zootaxa 2214: 1-28. https://doi.org/10.5281/zenodo.189912

Mengual X, Ståhls G, Rojo S (2008) First phylogeny of predatory flower flies (Diptera, Syrphidae, Syrphinae) using mitochondrial COI and nuclear 28S rRNA genes: conflict and congruence with the current tribal classification. Cladistics 24: 543-562. https://doi. org/10.1111/j.1096-0031.2008.00200.x

Mengual X, Thompson FC (2008) Palpada prietorum, a new flower fly from Colombia (Diptera: Syrphidae). Zootaxa 1742: 31-36. https://doi.org/10.5281/zenodo.181531

Mengual X, Thompson FC (2011) Carmine cochineal killers: the flower fly genus Eosalpingogaster Hull (Diptera: Syrphidae) revised. Systematic Entomology 36(4): 713-731. https:// doi.org/10.1111/j.1365-3113.2011.00588.x

Metz MA, Thompson FC (2001) A revision of the larger species of Toxomerus (Diptera: Syrphidae) with description of a new species. Studia Dipterologica 8: 225-256.

Miranda GF, Marshall SA, Skevington JH (2014) Revision of the genus Pelecinobaccha Shannon, description of Relictanum gen. nov., and redescription of Atylobaccha flukiella (Curran, 1941) (Diptera: Syrphidae). Zootaxa 3819(1): 1-154. http://doi.org/10.11646/ zootaxa.3819.1.1

Miranda GF, Skevington JH, Marshall SA, Kelso S (2016) The genus Ocyptamus Macquart (Diptera: Syrphidae): a molecular phylogenetic analysis. Arthropod Systematics and Phylogeny 74(2): 161-1976.

Mittermeier PR, Mittermeier CG (1997) Megadiversidad. Los países biológicamente más ricos del mundo. CEMEX y Agrupación Sierra Madre. México.

Mittermeier RA, Gil PR, Hoffman M, Pilgrim J, Brooks T, Mittermeier CG, Lamoreux J, da Fonseca GAB (2004) Hotspots revisited: Earth's biologically richest and most endangered terrestrial ecoregions. CEMEX, Conservation International, $392 \mathrm{pp}$.

Mittermeier RA, Mittermeier CG, Gil PR, da Fonseca GAB, Brooks T, Pilgrim J, Konstant WR (2005) Megadiversity: Earth's biologically wealthiest nations. CEMEX, Mexico, 501 pp.

Montoya AL (2016) Family Syrphidae. Zootaxa 4122: 457-537. http://doi.org/10.11646/ zootaxa.4122.1.39

Montoya AL, Pérez SP, Wolff M (2012) The diversity of flower flies (Diptera: Syrphidae) in Colombia and their Neotropical distribution. Neotropical entomology 41(1): 46-56. https:// doi.org/10.1007/s13744-012-0018-z

Morales MN, Marinoni L (2009) Cladistic analysis and taxonomic revision of the scutellaris group of Palpada Macquart (Diptera: Syrphidae). Invertebrate Systematics 23: 301-347. https://doi.org/10.1071/IS09006

Morales MN, Marinoni L (2017) Syrphidae. Taxonomic Catalog of the Brazilian Fauna. http:// fauna.jbrj.gov.br/

Moret P (2005) Los coleópteros Carabidae del páramo en los Andes del Ecuador. Sistemática, ecología y biogeografía. Museo de Zoología, Centro de Biodiversidad y Ambiente, Escuela de Biología. Pontificia Universidad Católica del Ecuador. Gruppo Editoriale Il Capitello, Italia, $307 \mathrm{pp}$. 
Myers N, Mittermeier RA, Mittermeier CG, da Fonseca GAB, Kent J (2000) Biodiversity hotspots for conservation priorities. Nature 403: 853-858. http://doi.org/10.1038/35002501 Nelson EH, Hogg BN, Mills NJ, Daane KM (2012) Syrphid flies suppress lettuce aphids. BioControl 57: 819-826. https://doi.org/10.1007/s10526-012-9457-z

Pape T, Thompson FC (Eds) (2013) Systema Dipterorum, version 1.5. http://www.diptera.org/ Peck SB (1994) Aerial dispersal of insects between and to islands in the Galápagos Archipelago, Ecuador. Annals of the Entomological Society of America 87: 218-224. http://doi. org/10.1093/aesa/87.2.218

Peck SB (1996) Origin and development of an insect fauna on a remote archipelago: The Galápagos Islands, Ecuador. In: Keast A, Miller SE (Eds) The origin and evolution of Pacific Island biotas, New Guinea to Eastern Polynesia: Patterns and processes. SPB Academic Publishing, Amsterdam, 91-122.

Peck SB, Heraty J, Landry B, Sinclair BJ (1998) Introduced insect fauna of an oceanic archipelago: the Galápagos Islands, Ecuador. American Entomologist 44: 218-237. http://doi. org/10.1093/ae/44.4.218

Pérez-Bańón C, Juan A, Petanidou T, Marcos-García MA, Crespo MB (2003) The reproductive ecology of Medicago citrina (Font Quer) Greuter (Leguminosae): a bee-pollinated plant in Mediterranean islands where bees are absent. Plant Systematics and Evolution 241: 29-46. http://doi.org/10.1007/s00606-003-0004-3

Pérez-Lachaud G, Jervis MA, Reemer M, Lachaud J-P (2014) An unusual, but not unexpected, evolutionary step taken by syrphid flies: the first record of true primary parasitoidism of ants by Microdontinae. Biological Journal of the Linnean Society 111: 462-472. http:// doi.org/10.1111/bij.12220

Pineda A, Marcos-García MA (2008) Introducing barley as aphid reservoir in sweet-pepper greenhouses: Effect on native and released hoverflies (Diptera, Syrphidae). European Journal of Entomology 105: 531-535. http://doi.org/10.14411/eje.2008.070

Piñas F, Manzano I (1997) Mariposas del Ecuador. Vol. I. Géneros. Quito, Pontificia Universidad Católica del Ecuador, 115 pp.

Piñas F, Manzano I (2003a) Mariposas del Ecuador. Vol. 16a. Saturniidae. Compañía de Jesús, Quito, 79 pp.

Pińas F, Manzano I (2003b) Mariposas del Ecuador. Vol. 21b. Arctiidae. Subfamilia: Ctenuchinae. Compañía de Jesús, Quito, 97 pp.

QGIS Development Team (2009) QGIS Geographic Information System. Open Source Geospatial Foundation. http://qgis.osgeo.org

Rasmussen C (2016) J. C. Bradley's narrative of the Cornell Entomological Expedition to South America (1919-1920): collecting localities and entomological travel details. Journal of History of Collections 28(1): 137-147. https://doi.org/10.1093/jhc/fhu074

Reemer M (2010) A second survey of Surinam Syrphidae (Diptera): Introduction and Syrphinae. Tijdschrift voor Entomologie 153: 163-196. https://doi.org/10.1163/22119434900000295

Reemer M (2013) Taxonomic exploration of Neotropical Microdontinae (Diptera: Syrphidae) mimicking stingless bees. Zootaxa 3697: 1-88. http://doi.org/10.11646/zootaxa.3697.1.1 
Reemer M (2014) A review of Microdontinae (Diptera: Syrphidae) of Surinam, with a key to the Neotropical genera. Tijdschrift voor Entomologie 157: 27-57. https://doi. org/10.1163/22119434-00002035

Reemer M (2016) Syrphidae (Diptera) of Surinam: Eristalinae and synthesis. Tijdschrift voor Entomologie 159: 97-142. https://doi.org/10.1163/22119434-00002035

Reemer M, Rotheray GE (2009) Pollen feeding larvae in the presumed predatory syrphine genus Toxomerus Macquart (Diptera, Syrphidae). Journal of Natural History 43: 939-949. http://dx.doi.org/10.1080/00222930802610576

Reemer M, Ståhls G (2013a) Generic revision and species classification of the Microdontinae (Diptera, Syrphidae). ZooKeys 288: 1-213. https://doi.org/10.3897/zookeys.288.4095

Reemer M, Ståhls G (2013b) Phylogenetic relationships of Microdontinae (Diptera: Syrphidae) based on molecular and morphological characters. Systematic Entomology 38: 661-688. http://dx.doi.org/10.1111/syen.12020

Ricarte A, Marcos-García MA, Hancock EG, Rotheray EG (2012) Revision of the New World genus Quichuana Knab, 1913 (Diptera: Syrphidae), including descriptions of 24 new species. Zoological Journal of the Linnean Society 166: 72-131. https://doi.org/10.1111/ j.1096-3642.2012.00842.x

Ricarte A, Marcos-García MA, Hancock EG, Rotheray GE (2015) Neotropical Copestylum Macquart (Diptera: Syrphidae) breeding in fruits and flowers, including 7 new species. PLoS ONE 10(11): e0142441. https://doi.org/10.1371/journal.pone.0142441

Ricarte A, Marcos-García MA, Moreno CE (2011) Assessing the effects of vegetation type on hoverfly (Diptera: Syrphidae) diversity in a Mediterranean landscape: implications for conservation. Journal Insect Conservation 15: 865-877. https://doi.org/10.1007/s10841011-9384-9

Rojo S, Gilbert F, Marcos-García MA, Nieto JM, Mier MP (2003) A world review of predatory hoverflies (Diptera, Syrphidae: Syrphinae) and their prey. Centro Iberoamericano de la Biodiversidad (CIBIO), Alicante, 278 pp.

Rondani C (1851) Dipterorum species aliquae in America aequatoriali collectae a Cajetano Osculati, observatae et distinctae novis breviter descriptis. Nuovi Annali delle Scienze Naturali. Bologna (3) 2(1850): 357-372.

Rotheray GE (1993) Colour guide to hoverfly larvae (Diptera, Syrphidae) in Britain and Europe. Dipterists Digest No. 9, England.

Rotheray GE, Gilbert F (1999) Phylogeny of Palaearctic Syrphidae (Diptera): evidence from larval stages. Zoological Journal of the Linnean Society London 127: 1-112. https://doi. org/10.1111/j.1096-3642.1999.tb01305.x

Rotheray GE, Gilbert F (2011) The natural history of hoverflies. Forrest Text, Ceredigion, 333 pp.

Rotheray GE, Hancock EG, Marcos-García MA (2007) Neotropical Copestylum (Diptera, Syrphidae) breeding in bromeliads (Bromeliaceae) including 22 new species. Zoological Journal of the Linnean Society 150: 267-317. https://doi.org/10.1111/j.1096-3642.2007.00288.x

Rotheray GE, Marcos-García MA, Hancock EG, Gilbert FS (2000) The systematic position of Alipumilio and Nausigaster based on early stages (Diptera, Syrphidae). Studia dipterologica 7(1): 133-144. 
Rotheray GE, Marcos-García MA, Hancock G, Pérez-Bañón C, Maier CT (2009) Neotropical Copestylum (Diptera, Syrphidae) breeding in Agavaceae and Cactaceae including seven new species. Zoological Journal of the Linnean Society 156(4): 697-749. https://doi. org/10.1111/j.1096-3642.2008.00503.x

Rotheray GE, Zumbado M, Hancock EG, Thompson FC (2000) Remarkable aquatic predators in the genus Ocyptamus (Diptera, Syrphidae). Studia Dipterologica 7: 385-398.

Salazar F, Donoso D (2014) El mundo de los invertebrados: en busca del número total de especies del Ecuador. Nuestra Ciencia 16: 37-40.

Schiner IR (1868) Diptera. In: Wüllerstorf-Urbair B von (Ed) Reise der österreichischen Fregatte Novara um die Erde in den Jahren 1857, 1858, 1859. Zoologischer Theil, Vol. 2, Abteilung 1, [Sect.]. BK Gerold's Sohn, Wien, 388 pp. [4 pls]

Schmidt MH, Thewes U, Thies C, Tscharntke T (2004) Aphid suppression by natural enemies in mulched cereals. Entomologia Experimentalis et Applicata 113: 87-93. https://doi. org/10.1111/j.0013-8703.2004.00205.x

Shannon RC (1927) A review of the South American two-winged flies of the family Syrphidae. Proceedings of the United States National Museum 70(9): 1-33. http://doi.org/10.5479/ si.00963801.70-2658.1

Sinclair BJ (2015) CDF Checklist of Galapagos flies. In: Bungartz F, Herrera H, Jaramill P, Tirado N, Jiménez-Uzcátegui G, Ruiz D, Guézou A, Ziemmeck F (Eds) Charles Darwin Foundation Galapagos Species checklist. Charles Darwin Foundation, Puerto Ayora, Galapagos, 1-52. http://checklists.datazone.darwinfoundation.org/terrestrial-invertebrates/diptera/

Sinclair BJ, Peck S (2002) An annotated checklist of the Diptera of Galápagos Archipelago (Ecuador), $54 \mathrm{pp}$.

Sinclair BJ, Thompson FC, Wyatt N (2016) The flower flies of the Galápagos Islands (Diptera: Syrphidae). Entomologist's Monthly Magazine 152: 79-96.

Smith F (1877) Account of the Zoological Collections made during the visit of H. M. S. "Petrel" to the Galapagos Islands. VIII. Hymenoptera and Diptera. Proceedings of the Zoological Society of London 45: 82-84.

Sommaggio D (1999) Syrphidae: can they be used as environmental bioindicators. Agriculture, Ecosystems and Environment 74: 343-356. http://doi.org/10.1016/S01678809(99)00042-0

Sommaggio D, Burgio G (2014) The use of Syrphidae as functional bioindicator to compare vineyards with different managements. Bulletin of Insectology 67(1): 147-156. http:// www.bulletinofinsectology.org/pdfarticles/vol67-2014-147-156sommaggio.pdf

Speight MCD (2016) Species accounts of European Syrphidae 2016. In: Speight MCD, Castella E, Sarthou JP, Vanappelghem C (Eds) Syrph the Net, the database of European Syrphidae (Diptera), Vol. 93. Syrph the Net publications, Dublin, 288 pp.

Speight M, Lucas J (1992) Liechtenstei Syrphidae (Diptera). Verhandlungen der ZoologischBotanischen Gesellschaft. Liechtenstein Sargans Werdenberg 19: 327-463.

Ssymank A, Kearns C (2009) Flies-pollinators on two wings. In: Ssymank A, Hamm A, VischerLeopold M (Eds) Caring for pollinators - safeguarding agro-biodiversity and wild plant diversity. Bundesamt für Naturschutz, Bonn, 39-52. 
Thompson FC (1972) A contribution to a generic revision of the Neotropical Milesinae (Diptera: Syrphidae). Arquivos de Zoologia 23(2): 73-215. https://repository.si.edu/handle/10088/17138

Thompson FC (1973) Review of the genus Sterphus Philippi (Diptera: Syrphidae). Part I. Entomologica Americana 46: 185-240. https://repository.si.edu/handle/10088/17139

Thompson FC (1991) The flower fly genus Ornidia (Diptera: Syrphidae). Proceedings of the Entomological Society of Washington 93: 249-262. https://repository.si.edu/handle/10088/17487

Thompson FC (1997) Revision of the Eristalis flower flies (Diptera: Syrphidae) of the Americas south of the United States. Proceedings of the Entomological Society of Washington 99: 209-237. https://repository.si.edu/handle/10088/17085

Thompson FC (1999) A key to the genera of the flower flies of the Neotropical Region including the descriptions of genera and species and a glossary of taxonomic terms. Contributions on Entomology, International 3: 319-378. https://repository.si.edu/handle/10088/17492

Thompson FC (2006) Primer Taller de Identificación de Syrphidae del Neotrópico. Universidad del Valle, Cali, Colombia, 618 pp.

Thompson FC (2012) Costarica Mengual \& Thompson, 2009 (Insecta: Diptera: Syrphidae) junior homonym of Costarica Kocak \& Kemal, 2008 (Insecta: Orthoptera): proposed replacement by Tiquicia nom. nov. Zootaxa 3360: 68. http://doi.org/10.11646/zootaxa.3360.1.3

Thompson FC (2013) Syrphidae. Systema Dipterorum, version 1.5, 13354 records. http:// www.diptera.org

Thompson FC (2015) What is Priomerus Macquart? A 180 year old mystery resolved (Diptera: Syrphidae). Entomologist's Monthly Magazine 151: 168.

Thompson FC, Thompson BJ (2007) A new Toxomerus species from Chile (Diptera: Syrphidae). Studia Dipterologica 13: 317-331. https://repository.si.edu/handle/10088/4385

Thompson FC, Zumbado MA (2002) Mesoamerican Mallota flower flies (Diptera: Syrphidae) with the description of four new species. Studia Dipterologica 9: 89-107. https://repository.si.edu/bitstream/handle/10088/17099/ent_FCT_93.pdf

Thompson FC, Rotheray GE, Zumbado M (2010) Family Syrphidae. In: Brown B, Borkent A, Cumming JM, Wood DM, Woodley NE, Zumbado MA (Eds) Manual of Central American Diptera, Vol. 2. NRC Press, Ottawa, 763-792.

Thompson FC, Thompson BJ, Fairman JE (2000) Only in Costa Rica: New Neotropical flower flies (Diptera: Syrphidae). Studia Dipterologica 7: 33-43. https://repository.si.edu/handle/10088/17493

Thompson FC, Vockeroth JR, Sedman YS (1976) Family Syrphidae. A catalogue of the Diptera of the American South of the United States 46: 1-195. https://doi.org/10.5962/bhl. title. 49898

Thomson CG (1869) Diptera, Species nova descripsit. In: K. Svenska Vetenskaps-Akademien, Kongliga svenska fregatten Eugenies resa omkring jorden. Pt. Zoologie, 1: Insekter, 443-614. Thormann B, Ahrens D, Marín Armijos D, Peters MK, Wagner T, Wägele JW (2016) Exploring the leaf beetle fauna (Coleoptera: Chrysomelidae) of an Ecuadorian mountain forest using DNA barcoding. PLoS ONE 11:e0148268. https://doi.org/10.1371/journal. pone. 0148268 
Tscharntke T, Klein AM, Kruess A, Steffan-Dewenter I, Thies C (2005) Landscape perspectives on agricultural intensification and biodiversity-ecosystem service management. Ecology Letters 8: 857-874. https://doi.org/10.1111/j.1461-0248.2005.00782.x

Ureña O, Hanson P (2010) A fly larva (Syrphidae: Ocyptamus) that preys on adult flies. Revista de Biología Tropical 58(4): 1157-1163. http://doi.org/10.15517/rbt.v58i4.5401

Vockeroth JR (1969) A revision of the genera of the Syrphini (Diptera, Syrphidae). Memoirs of the Entomological Society of Canada 62: 1-176. https://doi.org/10.4039/entm10162fv

Vockeroth JR (1992) The flower flies of the Subfamily Syrphinae of Canada, Alaska, and Greenland (Diptera: Syrphidae). The Insects and arachnids of Canada, part 18. Centre for Land and Biological Resources Research, Ottawa, 456 pp. http://www.esc-sec.ca/aafcmonographs/insects_and_arachnids_part_18.pdf

Walker F (1849) List of the specimens of dipterous insects in the collection of the British Museum. Part III. British Museum (Natural History), London, 485-687.

Weng JL, Rotheray GE (2008) Another non-predaceous syrphine flower fly (Diptera: Syrphidae): pollen feeding in the larva of Allograpta micrura. Studia Dipterologica 15(1): 245-258.

Zuijen MP, Nishida K (2011) Description of life history and immature stages of phytophagous flower fly, Allograpta zumbadoi Thompson (Diptera: Syrphidae: Syrphinae). Studia dipterologica 17: 37-51. 